\title{
Centrosome loss results in an unstable genome and
}

\section{malignant prostate tumors}

Mengdie Wang ${ }^{1}$, Raymond B. Nagle ${ }^{2}$, Beatrice S. Knudsen ${ }^{3}$, Anne E. Cress ${ }^{1, *}$ and Gregory C.

Rogers ${ }^{1, *}$

1 Department of Cellular and Molecular Medicine, University of Arizona Cancer Center, University of Arizona, Tucson, Arizona 85724, USA

2 Department of Pathology, University of Arizona Cancer Center, University of Arizona, Tucson, Arizona 85724, USA

3 Department of Pathology and Laboratory Medicine, Cedars Sinai Medical Center, Los Angeles, CA 90048, USA

Co-corresponding authors: Anne E. Cress, Gregory C. Rogers

cress@email.arizona.edu, gcrogers@email.arizona.edu

Running Title: Centrosome loss in prostate tumors 


\begin{abstract}
Localized, non-indolent prostate cancer $(\mathrm{PCa})$ is characterized by large-scale genomic rearrangements, aneuploidy, chromothripsis, and other forms of chromosomal instability (CIN), yet how this occurs remains unclear. A well-established mechanism of CIN is the overproduction of centrosomes, which promotes tumorigenesis in various mouse models. Therefore, we developed a single-cell assay for quantifying centrosomes in human prostate tissue. Surprisingly, centrosome loss - which has not been described in human cancer - was associated with PCa progression. By chemically or genetically inducing centrosome loss in nontumorigenic prostate epithelial cells, mitotic errors ensued, producing aneuploid and multinucleated cells. Strikingly, transient or chronic centrosome loss transformed prostate epithelial cells which produced highly proliferative and poorly differentiated malignant tumors in mice. Our findings suggest that centrosome loss could create a cellular crisis with oncogenic potential in prostate epithelial cells.
\end{abstract}




\section{Introduction}

Tumorigenic driver mutations, common in other cancers, have not been detected in primary prostate tumors [1-3]. Indeed, localized PCa contains an overall low burden of single nucleotide variants (SNVs), while metastatic PCa has multiple specific recurrently altered genes and pathways [3-7]. Instead, primary PCa displays nuclei with defects in size and DNA content, and enriched with large-scale genomic rearrangements, copy number variations (CNV), changes in DNA methylation patterns, chromothripsis, gene fusions and other forms of genomic instability [8-10]. By analyzing the clonal status of genomic lesions within prostate tumors, a model has been proposed whereby oncogenic genomic alterations occur in a few successive bursts [1]. Mutations accumulate slowly at first, and a subset of driver mutations occurs late in PCa development $[1,11]$. Thus, oncogenic events for localized PCa might include an unidentified mechanism, independent of specific driver mutations, to generate an early genomic crisis.

A genomic crisis could arise due to a change to centrosome numbers. Centrosomes are the major microtubule-organizing center in cells, giving rise to a variety of protein machines, including mitotic spindles [12]. At the centrosome core lies a centriole pair that serve as the duplicating elements of the organelle and recruit an organized shell of pericentriolar material possessing microtubule-nucleating activity $[13,14]$. Cells exert tight control over centrosome copy number because centrosome overduplication (known as 'amplification') or centrosome loss produce cellular dysfunction and disease $[15,16]$.

Centrosome amplification is recognized as a general feature of tumors, seen in several solid tumors and blood cancers, and correlates with advanced tumor grade, recurrence, and poor survival [17]. Excess centrosomes lead to assembly of transient multipolar spindles with incorrect chromosome attachments $[18,19]$. Consequently, anaphase lagging chromatids are 
produced, forming micronuclei susceptible to chromothripsis [20]. Induction of centrosome amplification in mice promotes tissue hyperplasia, and formation of solid tumors with focal CNV and whole chromosome aneuploidy [21-24].

In the opposite condition, vertebrate cells lacking centrosomes use an acentrosomal microtubule assembly pathway [25], and their proliferation is dependent on p53 status [26-28]. Acentrosomal spindles cause prolonged mitosis as well as mitotic errors including anaphase lagging chromatids, micronuclei, aneuploidy, and polyploidy [27-30]. Although centrosome amplification and loss have remarkably similar effects in promoting CIN, centrosome loss has not been reported in cancer.

Here, we show that human prostate tumors display significant centrosome loss. Moreover, centrosome loss in non-tumorigenic prostate epithelial cells results in genomic alterations and malignant mouse xenograft tumors. Our results suggest that centrosome loss is a cellular crisis in prostate cancer, potentially explaining the presence of large-scale genomic defects without specific recurrent mutations. 


\section{Results}

\section{Localized prostate tumors display significant centrosome loss with increasing grade}

Localized prostate cancer was analyzed for centrosome detection and quantitation using an in situ immunofluorescence microscopy assay developed by us [35]. Since a pair of centrioles form the centrosome core, antibodies were used against centriole protein CEP135 and the PCM protein $\gamma$-Tubulin; co-localization of CEP135 and $\gamma$-Tubulin foci confirmed our ability to detect bona fide centrosomes (Fig. S1). E-Cadherin staining demarcated epithelial cell borders.

Localized PCa shows elevated E-cadherin expression and, although no significant differences in E-Cadherin expression associate with higher Gleason scores [36], we used $\alpha 6$ integrin as an additional membrane marker.

Tissues were imaged in multiple z-sections, and although centrosomes are small, our approach reliably identified cell boundaries and maximized our ability to image entire cell volumes, allowing us to measure centrosomes within individual cells. Normal human prostate glands possess basal and luminal cell layers (Fig. 1a). CEP135 localizes to the proximal end of each centriole within the pair and, as expected, appeared as two distinct spots in the majority of basal and luminal cells (Fig. 1a, f). Each cell contained two CEP135 foci that could be positioned in different z-section planes or within the same focal section (Fig. 1a). Approximately $20 \%$ of cells in normal prostate glands contained no centrosomes (Fig. 1f), likely due to the known loss of cell content during tissue sectioning. Surprisingly, Gleason grade 3 PCa exhibited a significant increase in cells lacking centrosomes compared to cells of normal tissue. The proportion of cells devoid of centrosomes increased significantly with each increase of tumor grade: $52 \%$ for Gleason grade 4 and $73 \%$ for Gleason grade 5 . This pattern held true when centrosome numbers are counted using CEP135 or $\gamma$-Tubulin foci (Fig. 1b, c, and f). 
Importantly, centrosomes were readily observed in surrounding normal stromal cells, distinguished as cells lacking E-Cadherin and serving as an internal control (Fig. 1c, yellow arrowheads). Detection of centrosomes in stromal cells demonstrated that our assay can identify centrosomes in cells with small volumes within the same section.

As a further validation for our assay, we quantified centrosomes in triple-negative breast cancer (TNBC), which is known to display centrosome amplification [37]. Compared to normal mammary glands, we confirmed that TNBC contains a significantly higher fraction of cells with $>2$ CEP135 foci (24\%) (Fig. 1d-f). Since TNBCs contain low levels of differentiation markers and lack basal-apical polarity [38], our assay is effective regardless of cell polarity or differentiation status. Thus, unlike most epithelial cancers described to date, centrosomes are lost in localized PCa and in prostate tumors with higher Gleason scores.

\section{PCa cell lines show both centrosome amplification and loss}

We next compared centrosome numbers in primary and immortalized non-tumorigenic prostate epithelial cells as well as metastatic and neuroendocrine PCa-derived cell lines. Cells were immunostained for CEP135 and CENT3 (an additional centriole marker) or $\gamma$-Tubulin, and the numbers of foci per cell were measured. Most prostate epithelial cells (80\%), including PEC (primary) as well as PrEC, iPEC37, and RWPE1 (immortalized), contained a normal centrosome count (two CEP135 foci per cell) while few displayed centrosome loss (zero CEP135 foci per cell) or amplification ( $>2$ CEP135 foci per cell) (Fig. 2a-d, j). However, metastasis-derived PCa cell lines displayed either centrosome loss or amplification and could be sub-classified by their androgen receptor expression status. Androgen-responsive cell lines, LNCaP and VCaP, showed significant centrosome loss compared to RWPE1 cells (Fig. 2e, f, j). Similar loss of centrosomes 
in LNCaP cells was previously reported [39]. In contrast, castration-resistant DU145 and PC3 cells, and neuroendocrine H660 cells, displayed centrosome amplification (Fig. 2g-j), as previously described $[39,40]$. Thus, centrosome numbers during tumor progression may be dynamic. While a majority of primary prostate $\mathrm{PCa}$ in situ show centrosome loss, centrosome amplification appears in more clinically-aggressive variants of hormone-resistant metastatic cancer.

\section{Centrosome loss in prostate epithelial cells results in mitotic errors, chromosome fragmentation, and micronuclei}

We next sought to determine whether the centrosome loss in localized PCa could account for the abundant genomic alterations reported [5]. To test this, we used two immortalized prostate epithelial cell lines, RWPE1 and PrEC; both have been used as stable, benign in vitro models [33, 41-44] and contain normal centrosome numbers (Fig. 2j). We first induced centrosome loss chemically by treating cells with centrinone, a Polo-like kinase 4 (Plk4) inhibitor [28]. Plk4 is the master-regulator of centriole duplication [45, 46], and its inhibition causes progressive centrosome depletion in proliferating cell cultures [28]. Over $90 \%$ of cells contained either one or zero centrosomes after a 10-day treatment but reassembled centrosomes within two days after centrinone removal (Fig. 3a). As a control for off-targeting effects, we generated a centrinoneresistant RWPE1 line, designated RWPE1-Plk4 (G95L), by substituting Leu for Gly95 (G95L) within the endogenous Plk4 gene using CRISPR/Cas9 [28]. Centrinone had no effect on centrosome number in RWPE1-Plk4 (G95L) cells after 10 days of drug treatment (Fig. 3b).

We next determined how centrosome loss affected mitosis in prostate epithelial cells using cells stably expressing histone H2B-GFP and centriole marker CETN1-tdTomato. After a 
10-day mock or centrinone treatment, we identified live cells with and without centrosomes (based on the CETN1 signal) and imaged chromosomes as cells progressed through mitosis by time-lapse microscopy (Fig. 3c and Supplementary Videos 1,2). Whereas mock and DMSOtreated cells spent $\sim 102$ minutes in mitosis (defined here as nuclear envelope breakdown to telophase) (Fig. 3d, Supplementary Videos 3, 4), centrinone-treated cells lacking centrosomes spent 3-4 times longer in mitosis (Fig. 3d, Fig. S2a, Supplementary Videos 5, 6). Consequently, the mitotic index of centrosome-less cells increased 5-fold but remained similar to control frequencies when centrinone was applied to RWPE1-Plk4 (G95L) cells (Fig. S2b), demonstrating that the rise in mitotic index was due to Plk4 inactivation.

In centrinone-treated RWPE1 and PrEC cells, chromosomes clustered on disorganized spindles during a prolonged prometaphase (Fig. 3e, f, Supplementary Video 7), but eventually separated into two chromosome masses (Fig. 3c, Supplementary Videos 5-8). Lagging chromatids were present during anaphase (Fig. 3c, red arrowheads, Supplementary Videos 5, 6), which have been shown to form from abnormal merotelic kinetochore attachments in acentrosomal vertebrate spindles [30,47]. Strikingly, we detected several small chromosomes surrounding the central chromosome mass(es) (Fig. 3c, e) and that were inefficiently captured by spindles (Fig. 3f, yellow arrowheads). Immunostaining for the centromere protein CENP-C revealed these errant chromosomes lacked centromeres and, thus, are chromosome fragments (Fig. 3f, Supplementary Video 7). Mis-segregated chromosome fragments were enveloped into micronuclei during mitotic exit, causing an increase of micronuclei frequency that was not seen in RWPE1-Plk4 (G95L) cells (Fig. 3g and Fig. S2c). Cytokinesis failure was also frequent (30\%; 20 of 66 cells) in centrinone-treated cells (Fig. 3c, RWPE1), and continuous imaging revealed that $80 \%$ of these cells died soon after entering G1-phase. As an alternative to 
centrinone, we generated two Plk4- null clonal lines using CRISPR (Plk4KO-1 and Plk4KO-2)

(Fig. S2d, e); immunostaining for CEP135 and $\gamma$-Tubulin confirmed that both clones lacked centrosomes. Both Plk4KO-1 and KO-2 clones showed a significant increase in the frequency of micronuclei (Fig. 3g). Notably, 61\% of micronuclei in centrinone-treated RWPE1 cells lacked CENP-C (Fig. 3h), suggesting that most micronuclei formed from chromosome fragments.

Lastly, we examined whether centrosome loss also generates micronuclei in 3dimensional (3D) cultured spheroids that approximate normal prostate glands [48]. Similar to 2D cultures, centrinone-treatment produced spheroids with numerous micronuclei (Fig. 3i, arrowheads). Taken together, our findings suggest that centrosome loss in cultured prostate epithelial cells induces phenotypes known to promote CIN.

Centrosome loss produces abnormal nuclear envelopes and increased multinucleate cells in PCa tissue

We next imaged chromosomes of cultured cells exiting mitosis by time-lapse microscopy. Interestingly, we observed mitotic cells that formed multi-nucleated daughter cells as well as abnormally-shaped, multi-lobed nuclei in spite of having no apparent defects in anaphase chromosome segregation or cytokinesis (Fig. 4a, Supplementary Videos 9, 10).

Since cells of early PCa contain nuclei with morphological defects [9], we analyzed whether centrosome loss results in defects in nuclear morphology by immunostaining cells for nuclear lamina proteins, Lamin A/C or Emerin. Plk4KO PrEC cells or centrinone-treated wildtype PrEC (or RWPE1) cells displayed a significant 3-9 fold increase in multinucleation frequency compared to control cells (Fig. 4b-d). Nuclear envelopes in acentrosomal cells 
frequently displayed invaginations (Fig. 4b, arrowheads), irregularities commonly seen in cancer cells [49].

Next, we examined whether similar nuclear defects were present in localized human PCa tissue by immunostaining for Lamin A/C or Emerin (Fig. 4e). Compared to cells from normal prostate glands, Gleason grade 3 PCa showed a higher average frequency of multinucleated cells but this was not significantly different (Fig. 4f). Gleason grade 4 PCa had an almost 4-fold increase of multinucleated cells (Fig. 4e, 1 and 2, and f) and micronuclei were detected (Fig. 4e, 2' arrowhead). Co-staining for CEP135 and DNA revealed that approximately $70 \%$ of multinucleated cells displayed complete centrosome loss (Fig. 4g, h). Taken together, our findings suggest that centrosome loss promotes multinucleation and defects in nuclear envelope morphology in cultured cells, and these defects are also present in higher grade PCa. Although it is unclear how these defects arose, it is noteworthy that normal nuclear envelope shape and the efficiency of nuclear reformation rely on the suppression of microtubule growth near chromatin, which happens to be the primary site of microtubule assembly in mitotic cells lacking centrosomes $[25,50]$.

\section{Centrosome loss results in aneuploidy and chromosomal rearrangements}

Localized PCa displays numerical and structural CIN [51]. Since centrosome loss in dividing prostate epithelial cells promotes mitotic errors, we evaluated if centrosome loss could induce aneuploidy by measuringchromosome numbers from chromosome spreads of mitotic RWPE1 cells (Fig. 5a, b). Whereas mock and DMSO-treated cells had a near diploid average chromosome number (mock cells: average $=50$; DMSO cells: 48) as previously reported [41], centrinone treatment induced aneuploidy, significantly increasing the average chromosome 
number to 69 (Fig. 5b). Chromosome fragments were also detected (Fig. 5a, arrowheads), consistent with our live-cell imaging data and the chromosome fragmentation reported in cells with micronuclei [20]. Flow cytometry confirmed that centrinone induced aneuploidy, displaying a 6-fold increase in the $>4 \mathrm{~N}$ population (Fig. 5c). DNA profiles of RWPE1-Plk4 (G95L) cells (with or without centrinone) remained similar to DMSO-control cells (Fig. 5c), suggesting that changes in chromosome number were due to centrosome loss.

Using spectral karyotyping (SKY), we next examined which specific chromosome exceeded diploidy in aneuploid, acentrosomal RWPE1 cells. Cells without centrosomes have altered chromosome copy numbers (gains and losses) compared to control cells, but primarily showed gains not specific to any particular chromosome (Fig. 5d-g). Cells that were stimulated to grow (with FBS) and centrinone-treated to deplete their centrosomes had a sharply increased chromosome number compared to FBS-mock treated cells whose karyotype was consistent with a previous report (Fig. e) [41]. The frequency of near tetraploid karyotypes ( $>80$ chromosomes) also increased by $30 \%$ after centrinone treatment, suggesting that cytokinesis failure likely contributed to some karyotypes. Furthermore, both RWPE1 cells and PrEC cells showed chromosomal structural rearrangements after centrinone treatment (Fig. S3a, b). Thus, instead of specific mutations, centrosome loss appears to generate gross genomic abnormalities.

\section{Centrosome loss can stimulate malignant xenograft tumor formation}

Our findings suggest that centrosome loss is a relatively early occurrence in localized PCa, producing CIN. We next determined if centrosome depleted non-tumorigenic prostate cells could generate xenograft tumors. PrEC-Plk4KO clones (clones which proliferate while chronically acentrosomal) were injected subcutaneously into male immunodeficient NSG mice 
and examined for tumor formation weekly for approximately 16 weeks. Parental PrEC cells did not form tumors (Fig. 6a), consistent with a previous study [33]. Tumors were not detected by palpation of live mice injected with the PrEC-Plk4KO-1 or 2 cell lines. However, after these mice were sacrificed on day 112 , tumors were found at $25 \%$ of the Plk4KO injection sites (Fig. 6a). The tumors were poorly differentiated and malignant as determined by histological examination. Indeed, tumors from both clones invaded into the surrounding adipose and muscle tissue (Fig. 6b). Notably, these tumors contained mitotic figures (Fig. 6c, red arrowhead), enlarged nuclei (green arrowhead), and multinucleated cells (yellow arrowheads), suggesting that tumor cells experience $\mathrm{CIN}$ and had histological characteristics of aggressive cancer.

Although centrosome depletion was sufficient to generate tumors, Plk4KO cells likely experience a continuous, high rate of CIN due to their acentrosomal state, which may explain the slow growth and hence small size of these tumors. To test this, we used an alternative to chronic centrosome absence by transiently depleting centrosomes with centrinone for several days, thereby potentially inducing a burst of CIN in the parental PrEC line. After removal of the drug, centrosomes reappeared within the cell population, with the expectation that the potentially altered genomes would then be relatively stably-maintained and passed to progeny cells (outlined in Fig. 6d).. Accordingly, PrEC cells were treated with centrinone for 15 days ( $>90 \%$ of cells contained one or zero centrioles by day 10; Fig. 3a), followed by monoclonal expansion in normal culture media without centrinone. Using this approach, three of the ten generated clones (CTN-1, 6, and 9) displayed elevated MYC and ERG levels compared to the original PrEC cells (Fig. S4a, b). We selected these three lines for xenograft testing because elevated levels of MYC and ERG correlate with PCa progression $[52,53]$. CTN clones were genomically verified to be derived from PrEC using a STR profile. All CTN clones reestablished normal centrosome 
numbers after removal of the drug, similar to parental PrEC cells (Fig. S4c). Importantly, although the three CTN clones had normal centrosome numbers, they continued to display hallmarks of CIN and showed a significant, elevated frequency of micronuclei (Fig. S4d). All three strains inherited CIN from centrinone treatment, displaying dramatic deviations in chromosome number compared to the parental line; CTN-1 and CTN-6 showed chromosome loss, while CTN-9 had chromosome gain (Fig. S4e). Chromosomal rearrangements were also observed in CTN-9 by SKY analysis (Fig. S3b and S5a, b).

Next, the CTN clones were subcutaneously injected into male immunodeficient NSG mice to evaluate their tumorigenicity. As observed with the parental line, mice injected with CTN-1 did not form tumors ( 0 of 12) (Figs. 6e, f). Strikingly however, two CTN clones (CTN-6, 3 of 12; CTN-9, 9 of 12) formed large, visible tumors at the injection sites; the CTN-9 tumors developed much sooner than CTN-6 (80 days versus $\sim 100$ days, respectively) (Fig. 6e, f). Histological examination of all tumors revealed that they were similar regardless of the specific injected clone, forming highly proliferative malignant tumors with poorly differentiated epithelial glands (Fig. 6g). Similar to PrEC-Plk4KO clones, both CTN clones formed tumors that invaded the surrounding smooth muscle and adipose tissue (Fig. 6h, arrowheads). Thus, transient centrosome elimination can transform cells, enabling them to form malignant tumors.

We further characterized the CTN tumors by immunostaining tissue sections with a panel of epithelial, mesenchymal, and secretory markers (Fig. S5c, d). CTN tumors maintained epithelial proteins including CD49f and CD44 but were negative for E-cadherin, CK5/14, CK8/18, CK14-16, 19, as well as luminal (FOXA1), neuroendocrine (synaptophysin), and mesenchymal (vimentin, desmin) markers (Fig. S5c, d). Tumors were highly secretory and 
stained positive for mucin and hyaluronic acid (HA) (Fig. S5c, d), a feature of poorly differentiated malignant PCa [54].

Lastly, we measured centrosome numbers within all 12 tumors using our in situ centrosome-counting assay. As in PCa tissue, we identified individual cells that clearly contained CEP135-labeled centrosomes (Fig. 6i). Dividing cells were also observed and formed mitotic spindles regardless of their centrosome status (Fig. 6j). Surprisingly, many tumor cells displayed centrosome loss, in some CTN-9 tumors, the percentage of cells with zero centrosomes exceeded $70 \%$ (Fig. 6k). Thus, transient removal of centrosomes was sufficient to induce tumor formation in a subset of immortalized prostate epithelial PrEC cells. During tumorigenesis, centrosomes were lost in a large population of cells resident at the initial injection site, resembling the phenomenon we observed in primary prostate carcinoma. 


\section{DISCUSSION}

In lieu of signature driver mutations that distinguish many cancers, genomic instability itself may provide the primary oncogenic force behind prostate tumorigenesis. Localized PCa show widespread genomic rearrangements, including chromothripsis ( $20 \%$ of tumors) [5]. Moreover, genomic alterations occur in a few successive bursts, likely initiated by a genomic crisis, leading to a proposed punctuated-progression model of prostate tumor evolution $[1,11]$. Extensive studies have been performed to understand the complexity of genomic instability in prostate tumors and our findings point to centrosome loss as a potential origin of genomic crisis.

Centrosome loss is prevalent in localized primary prostate carcinoma, becoming more pronounced in higher Gleason grade tumors. Centrosome loss also promotes aneuploidy, chromosome fragmentation, micronuclei, and other forms of CIN. Thus, centrosome loss, in principle, may account for the wide range of genomic hallmarks assigned to localized PCa. Transient centrosome loss produced much larger solid tumors, suggesting that bursts of genomic crisis may be more suitable for sustained proliferation. Centrosome amplification has been recognized by many as a cancer hallmark with the capacity to promote oncogenesis [15]. It is surprising that the opposite situation, the absence of centrosomes in proliferating tumor cells, has not been described before in human cancer. Similar to multi-centrosomal/multipolar spindles, acentrosomal spindles display errors in positioning chromosomes and high rates of CIN [30]. We observed that centrosome depletion in human epithelial prostate cells is transformative, enabling the previously non-tumorigenic cells to form malignant tumors in mice. Future studies should determine whether centrosome loss is a phenomenon restricted to PCa.

If centrosome loss is a driver of genomic instability in early prostate tumors, what then causes this organelle to disappear? In general, little is known about the mechanisms of 
centrosome elimination. However, centrosomes are normally inactivated or lost during specific developmental stages in different animals [55]. For example, in the Drosophila female germline, centrioles disassemble when PCM is down-regulated; this mechanism contributes to centriole elimination in the oocyte [56]. In contrast, pericentrin, a PCM component, accumulates in prostate carcinoma [57], suggesting that changes in PCM levels may not be a mechanism underlying centrosome disappearance in primary prostate tumors. Centrosome loss may be due to other factors such as age, inflammation, hypoxia, other environmental influences, or a combination of events. Determining the root cause of centrosome disappearance will be an important line of investigation. Indeed, an unidentified environmental factor(s) might be responsible for the loss of centrosomes from the xenograft tumors that arose from injections of cells that had been transiently centrosome-depleted but contained a normal centrosome number at the time of injection.

Although centrosome loss occurs in primary prostate tumors, TNBC displays centrosome amplification [38], as do metastatic-derived androgen unresponsive PCa cell lines and neuroendocrine H660 PCa cells. Possibly, centrosome numbers fluctuate during prostate tumor evolution. Indeed, centrosome loss may be an early 'driver' event and, through further genomic instability, gives rise to cells with genotypes that trigger centrosome amplification, paving the way to malignancy. Notably, centrosome amplification promotes an invasive cellular behavior $[58,59]$. Additional studies will be needed to determine when centrosome loss occurs in PCa. Moreover, we found a possible relationship between centrosome duplication and hormone receptor status. Specifically, clinically-aggressive variants of hormone-resistant metastatic cancer show centrosome amplification, raising the possibility that androgen receptor signaling might regulate centrosome duplication, which should be explored. For PCa, this is significant 
because loss of androgen signaling and gain of neuorendrocine features are indicative of increased lethality. In addition, changes in centrosome number could be used as a predictive biomarker for aggressive (high risk) disease and patient survival if it were established that centrosome numbers vary in tumors of different malignancies, particular those that are metastatic and recurrent. 


\section{Materials and Methods}

\section{Cell culture}

RWPE1 (ATCC \#CRL- 11609TM), PEC, and iPEC37 (C. Miranti, University of Arizona) cells were grown in Keratinocyte Serum Free Medium (K-SFM; ThermoFisher) with $0.05 \mathrm{mg} / \mathrm{ml}$ BPE, $5 \mathrm{ng} / \mathrm{ml} \mathrm{EGF}$ and penicillin/streptomycin/amphotericin $\left(5 \% \mathrm{CO}_{2}\right.$ at $\left.37^{\circ} \mathrm{C}\right)$. $\mathrm{PEC}$ was derived from prostatectomy specimens and verified to be free of stromal contamination $[31,32]$. Immortalized human PrEC cells (W. Hahn, MIT) [33] were cultured in Iscove's Modified Dulbecco's Medium (IMDM; ThermoFisher) with 10\% FBS. iPEC37 are PEC cells immortalized with HPV E6/E7 and hTERT [33]. LNCaP (ATCC C\#RL-1740), VCaP (ATCC \#CRL-2876), and PC3 (ATCC \#CRL-1435) were cultured in RPMI 1640 (ThermoFisher) with 10\% FBS, DU145 (ATCC \#HTB-81) in IMDM, and NCI-H660 (ATCC \#CRL-5813) in RPMI1640 media (supplemented with $0.005 \mathrm{mg} / \mathrm{ml}$ insulin, $0.01 \mathrm{mg} / \mathrm{ml}$ transferrin, $30 \mathrm{nM}$ sodium selenite, $10 \mathrm{nM}$ hydrocortisone, $10 \mathrm{nM}$ beta-estradiol, $2 \mathrm{mM} \mathrm{L-glutamine,} \mathrm{and} \mathrm{5 \%} \mathrm{FBS.} \mathrm{Cell}$ identities were verified using genomic probes [34]. For RWPE1 cultured in KSFM, centrinone was used at $100 \mathrm{nM}$, but $300 \mathrm{nM}$ for all cells cultured in FBS. Cells were serum starved for 48 hrs before addition of $100 \mathrm{nM}$ DHT.

\section{Stable lines and CRISPR/Cas9}

Lentiviral plasmid (pLenti6) and packaging vectors (pMD2.G/psPAX2; Addgene \#12259 and 12260) were used to generate H2B-GFP stable expressing cells. Virus was harvested from HEK293 cells at $24-48 \mathrm{hrs}$ post-transfection, filtered, and added to media with $8 \mu \mathrm{g} / \mathrm{ml}$ polybrene. Stable lines were selected with $500 \mathrm{ng} / \mathrm{ml}$ puromycin after 2 weeks. RWPE1 lines were then transduced with centrin1/CETN1)-tdTomato lentivirus (pLentiLox CMV; V. Bautch, University 
of North Carolina). The Alt-R CRISPR/Cas9 system (IDT) with Plk4 gRNA

(GGAAGCTGAGTGTTAAGTTC) was used to generate Plk4(G95L) [31]. The RNP complex of Plk4-crRNA, CRISPR-Cas9 tracrRNA (IDT \#1072532), and Cas9 were transfected into cells using a Nucleofector 2b (Amaxa). Repair template plasmid (pY181) (K. Oegema, University of California San Diego) and packaging plasmids (pHelper/pAAV-RC) were transfected into HEK293T cells using Lipofectamine300. Virus was harvested $48 \mathrm{hrs}$ post-transfection, filtered, and added to media for $16 \mathrm{hrs}$. Cells were selected in 96-well plates for two weeks in $0.5 \mathrm{mg} / \mathrm{ml}$ G418, and homozygous G95L knock-in clones were identified by PCR and sequencing of genomic DNA. UACC Genome Editing Facility generated Plk4 homozygous knock-out PrEC cells. Double-strand break sites flanking the Plk4 transcriptional unit $(4: 127,879,990$ and 4:127,909,266) were targeted with guide sequences 5-AACTACGGAATAAGCGGTAG-3 and 3-TCTCGCCAACTTAATTAAGG-5. Cells were transfected with Cas9, crRNAs, and tracrRNA using Lipofectamine. Two days post-transfection, cutting efficiency was estimated using a T7 endonuclease assay employing PCR primers flanking the predicted ligation-junction product. Single cells were deposited in 96-well plates using the UACC Flow Cytometry Shared Resource and colonies were screened by PCR. Clones that were negative for two fragments internal to the targeted deletion but positive for ligation-junction fragment were potentially homozygous for the deletion. Absence of Plk4 RNA was confirmed by rt-PCR.

\section{Microscopy}

For IF, cells were cultured on coverslips, washed in PBS, and fixed in ice-cold methanol for 10 mins. After a 5 min rehydration in PBS, cells were washed in Buffer-A (PBS, 0.5\% Triton X100) for 5 min, blocking Buffer-B (PBS, 5\% NGS, 0.1\% Triton X-100) for $30 \mathrm{~min}$, and 
incubated with primary antibodies in Buffer-B for $1 \mathrm{hr}$ at room temperature. Next, cells were washed 3 times in Buffer-A, incubated with secondary antibodies and Hoechst33342 for $1 \mathrm{hr}$ at room temperature, washed 3 times in Buffer-A ( $5 \mathrm{~min}$ ), and mounted in 0.1M n-propyl gallate, PBS, 90\% (by volume) glycerol. For centromere immunostaining, cells were rinsed in PHEM (60 mM PIPES, $25 \mathrm{mM}$ HEPES, $10 \mathrm{mM}$ EGTA, $4 \mathrm{mM} \mathrm{MgSO}_{4} \cdot 7 \mathrm{H}_{2} 0$ ), permeabilized with $0.5 \%$ Triton X-100 in PHEM for 4 min, and fixed with 4\% paraformaldehyde in PHEM for 20 min at room temperature.

For metaphase spreads, cells were treated with $0.06 \mu \mathrm{g} / \mathrm{ml}$ colcemid overnight, trypsinized, resuspended in Buffer-M (40 mM KCl, 20 mM HEPES, $0.5 \mathrm{mM}$ EGTA, and $9 \mathrm{mM}$ $\mathrm{NaOH}$ ), and incubated for $50 \mathrm{~min}$ at $37^{\circ} \mathrm{C}$. Next, cells were fixed in methanol:acetic acid (3:1), pelleted, and washed in fixative before dropped onto a pre-cleaned coverglass. Cells were dried on slides for 3 days and stained with Hoechst33342. Spectral karyotyping analysis was performed by the Roswell Park Cancer Institute Cytogenetics SKY Core Laboratory. Formalinfixed de-identified human cancer tissues were processed for detection of centrosomes using our published procedure [35]. Specimens were imaged using a DeltaVision Core equipped with an Olympus IX71 microscope, 60X objective (1.42NA), and a cooled charge-coupled CoolSNAPHQ2 camera. For time-lapse microscopy, cells were grown in 8-well Lab-Tek chambered coverglass coated with anti- $\beta 1$ integrin/AIIB2 antibody to inhibit cell migration; images were acquired every 5 min with $4 \mu \mathrm{m}$ z-section thickness for 24 hrs. Images acquired with SoftWoRx v1.2.

Antibodies/stains used include: anti-CEP135 (cells-1:500; tissue-1:100; Abcam ab75005), anti- $\gamma$-tubulin (cells-1:500; tissue-1:100; Sigma Aldrich GTU-88), anti-ECadherin/M168 (1:100; Abcam ab76055), anti-Centrin-3/3E6 (1:500; Abnova H00001070- 
M01), anti-phospho-Histone H3 (1:1000; Cell Signaling Technology \#9706S), 7-AAD Staining Solution (1:50; BioLegend \#420404), Alexa Fluor ${ }^{\mathrm{TM}} 647$ Phalloidin (1:40; ThermoFisher A22287), anti- $\alpha$-tubulin (1:1500; Sigma Aldrich DM1A), anti-CENP-C (1:1000; MBL International PD030), anti-LaminA/C (1:100; Santa Cruz Biotechnology sc-6215), anti-Emerin (1:100; Sigma-Aldrich HPA000609), anti- $\beta 1$ integrin (1:1000, DSHB AIIB2), anti- $\alpha 6$ integrin/CD49f (1:100; produced in our lab), anti-CD44 (1:100; ThermoFisher \#156-3C11), antiN-Cadherin (1:100; Santa Cruz Biotechnology sc-8424), anti-CK5/14 (1:100; produced in our lab), anti-CK18 (1:100; Abcam EPR1626), anti-Vimentin (1:100; Cell Signaling Technology D21H3), anti-Desmin (1:100; Atlas Antibodies HPA018803), Hyaluronic Acid-Binding Protein (1:100; Sigma-Aldrich \#385911), anti-cMyc (1:50-100; Cell Signaling Technology D3N8F), anti-FOXA1(1:100; Abcam EPR10881), anti-Synaptophysin (1:100; Sigma-Aldrich MRQ-40), anti-laminin-332 (1:200; Abcam ab14509), anti-ITGB4 (1:100; Abcam ab110167), and anti-p63 (1:100; biobyt orb214808). Secondary antibodies (Jackson Immunoresearch) were used at $1: 1500$.

\section{Animal studies}

Mouse experiments were conducted with animal care and committee approval using the Experimental Mouse Shared Service. $10 \times 10^{6}$ prostate epithelial cells were mixed with an equal number of non-tumorigenic fibroblasts (WPMY-1) in $0.1 \mathrm{ml}$ growth factor reduced matrigel and injected subcutaneously into male NOD/SCID/IL2R $\gamma^{\text {null }}$ (NSG) mice (Jackson Laboratories); three mice per treatment group. Tumor growth was measured 2 times/week and tumor volume estimated according to the formula: $\left[(\text { width })^{2} \mathrm{x}\right.$ length $] / 2$. Animals were terminated by $\mathrm{CO}_{2}$ when total tumor reached $2000 \mathrm{~mm}^{3}$. 


\section{Statistical Analysis}

The statistical significance of differences in average measurements was evaluated using unpaired t-tests (GraphPad Prism 6.0). Means are significantly different if $\mathrm{P}<0.05$. In figures, “*” indicates $0.05>\mathrm{P} \geq 0.01$; “**” $0.01>\mathrm{P} \geq 0.001$; “***” $0.001>\mathrm{P} \geq 0.0001$; “****” $0.0001>\mathrm{P}$, and not significant " $\mathrm{ns}$ " indicates $\mathrm{P} \geq 0.05$ for indicated pairwise comparisons. Error bars in all figures indicate standard deviation (SD). 
Acknowledgements We thank K. Oegema for providing centrinone and CRISPR constructs, D. Pellman for histone-H2B construct, V. Bautch for centrin1 construct, W. Hahn for PrEC cells, C. Miranti for iPEC37 cells, and N. Ellis and J. DeLuca for technical advice. Tissue samples were obtained from the Prostate Cancer Biorepository Network (PCBN) (DoD Prostate Cancer Research Program Award No W81XWH-14-2-0182, W81XWH-14-2-0183, W81XWH-14-20185, W81XWH-14-2-0186, and W81XWH-15-2-0062). This work was supported by NIH grants NCI P30 CA23074, R01GM110166 and R01GM126035 to G.C.R., R01CA159406 to A.E.C. and Tim and Diane Bowden Cancer Biology Research Fellowship to M.W.

\section{Compliance with ethical standards}

Conflict of interest The authors declare that they have no conflict of interest. 


\section{References}

1. Baca SC, Prandi D, Lawrence MS, Mosquera JM, Romanel A, Drier Y, et al. Punctuated evolution of prostate cancer genomes. Cell. 2013;153:666-677.

2. Boutros PC, Fraser M, Harding NJ, de Borja R, Trudel D, Lalonde E, et al. Spatial genomic heterogeneity within localized, multifocal prostate cancer. Nat Genet. 2015;47:736-745.

3. Armenia J, Wankowicz SAM, Liu D, Gao J, Kundra R, Reznik E, et al. The long tail of oncogenic drivers in prostate cancer. Nat Genet. 2008;50:645-651.

4. Barbieri CE, Baca SC, Lawrence MS, Demichelis F, Blattner M, Theurillat JP, et al. Exome sequencing identifies recurrent SPOP, FOXA1 and MED12 mutations in prostate cancer. Nat Genet. 2012;44:685-689.

5. Fraser M, Sabelnykova VY, Yamaguchi TN, Heisler LE, Livingstone J, Huang V, et al. Genomic hallmarks of localized, non-indolent prostate cancer. Nature. 2017;541:359-364.

6. Robinson DR, Wu YM, Lonigro RJ, Vats P, Cobain E, Everett J, et al. Integrative clinical genomics of metastatic cancer. Nature. 2017;548:297-330.

7. Wu YM, Cieslik M, Lonigro RJ, Vats P, Reimers MA, Cao X, et al. Inactivation of CDK12 delineates a distinct immunogenic class of advanced prostate cancer. Cell. 2018;173:17701782. 
8. Cancer Genome Atlas Research Network. The molecular taxonomy of primary prostate cancer. Cell. 2015;163:1011-1025.

9. Petein M, Michel P, van Velthoven R, Pasteels JL, Brawer MK, Davis JR, et al. Morphonuclear relationship between prostatic intraepithelial neoplasia and cancers as assessed by digital cell image analysis. Am J Clin Pathol. 1991;96:628-634.

10. Tomlins SA, Rhodes DR, Perner S, Dhanasekaran SM, Mehra R, Sun XW, et al. Recurrent fusion of TMPRSS2 and ETS transcription factor genes in prostate cancer. Science. 2005;310:644-648.

11. Espiritu SMG, Liu LY, Rubanova Y, Bhandari V, Holgersen EM, Szyca LM, et al. The evolutionary landscape of localized prostate cancers drives clinical aggression. Cell. 2018;173:1003-1013.

12. Conduit PT, Wainman A, Raff JW. Centrosome function and assembly in animal cells. Nat Rev Mol Cell Biol. 2015;16:611-624.

13. Bettencourt-Dias M, Glover DM. Centrosome biogenesis and function: centrosomics brings new understanding. Nat Rev Mol Cell Biol. 2007;8:451-463.

14. Lawo S, Hasegan M, Gupta GD, Pelletier L. Subdiffraction imaging of centrosomes reveals 
higher-order organizational features of pericentriolar material. Nat Cell Biol. 2012;14:11481158.

15. Nigg EA, Holland AJ. Once and only once: mechanisms of centriole duplication and their deregulation in disease. Nat Rev Mol Cell Biol. 2018;19:297-312.

16. Godinho SA, Pellman D. Causes and consequences of centrosome abnormalities in cancer. Philos Trans R Soc Lond B Biol Sci. 2014;369.

17. Chan JY. A clinical overview of centrosome amplification in human cancers. Int J Biol Sci. 2011;7:1122-1144.

18. Ganem NJ, Godinho SA, Pellman D. A mechanism linking extra centrosomes to chromosomal instability. Nature. 2009;460:278-282.

19. Silkworth WT, Nardi IK, Scholl LM, Cimini D. Multipolar spindle pole coalescence is a major source of kinetochore mis-attachment and chromosome mis-segregation in cancer cells. PLoS One. 2009;4:e6564.

20. Crasta K, Ganem NJ, Dagher R, Lantermann AB, Ivanova EV, Pan Y, et al. DNA breaks and chromosome pulverization from errors in mitosis. Nature. 2012;482:53-58.

21. Coelho PA, Bury L, Shahbazi MN, Liakath-Ali K, Tate PH, Wormald S, et al. Over- 
expression of Plk4 induces centrosome amplification, loss of primary cilia and associated tissue hyperplasia in the mouse. Open Biol. 2015;5:150209.

22. Levine MS, Bakker B, Boeckx B, Moyett J, Lu J, Vitre B, et al. Centrosome amplification Is sufficient to promote spontaneous tumorigenesis in mammals. Dev Cell. 2017;40:313-322.

23. Sercin O, Larsimont JC, Karambelas AE, Marthiens V, Moers V, Boeckx B, et al. Transient PLK4 overexpression accelerates tumorigenesis in p53-deficient epidermis. Nat Cell Biol. 2016;18:100-110.

24. Vitre B, Holland AJ, Kulukian A, Shoshani O, Hirai M, Wang Y, et al. Chronic centrosome amplification without tumorigenesis. Proc Natl Acad Sci USA. 2015;112:E6321-6330.

25. Meunier S, Vernos I. Acentrosomal microtubule assembly in mitosis: the where, when, and how. Trends Cell Biol. 2016;26:80-87.

26. Bazzi H, Anderson KV. Acentriolar mitosis activates a p53-dependent apoptosis pathway in the mouse embryo. Proc Natl Acad Sci USA. 2014;111:E1491-500.

27. Lambrus BG, Uetake Y, Clutario KM, Daggubati V, Snyder M, Sluder G, et al. p53 protects against genome instability following centriole duplication failure. J Cell Biol. 2015;210:63-77.

28. Wong YL, Anzola JV, Davis RL, Yoon M, Motamedi A, Kroll A, et al. Cell biology. 
Reversible centriole depletion with an inhibitor of Polo-like kinase 4. Science. 2015;348:11551160.

29. Khodjakov A, Rieder CL. Centrosomes enhance the fidelity of cytokinesis in vertebrates and are required for cell cycle progression. J Cell Biol. 2001;153:237-242.

30. Sir JH, Putz M, Daly O, Morrison CG, Dunning M, Kilmartin JV, et al. Loss of centrioles causes chromosomal instability in vertebrate somatic cells. J Cell Biol. 2013;203:747-756.

57. Edick MJ, Tesfay L, Lamb LE, Knudsen BS, Miranti CK. Inhibition of integrin-mediated crosstalk with epidermal growth factor receptor/Erk or Src signaling pathways in autophagic prostate epithelial cells induces caspase-independent death. Mol Biol Cell. 2007;18:2481-2490.

58. Gmyrek GA, Walburg M, Webb CP, Yu HM, You X, Vaughan ED, et al. Normal and malignant prostate epithelial cells differ in their response to hepatocyte growth factor/scatter factor. Am J Pathol. 2001;159:579-590.

37. Berger R, Febbo PG, Majumder PK, Zhao JJ, Mukherjee S, Signoretti S, et al. Androgeninduced differentiation and tumorigenicity of human prostate epithelial cells. Cancer Res. 2004;64:8867-8875.

59. van Bokhoven A, Varella-Garcia M, Korch C, Hessels D, Miller GJ. Widely used prostate carcinoma cell lines share common origins. Prostate. 2001;47:36-51. 
31. Wang, M, Knudsen, BS, Nagle RB, Rogers GC, Cress AE. A method to quantify centrosomes at single-cell level in human normal and cancer tissue. Mol Biol Cell. 2019;doi:10.1091/mcb.E18-10-0651.

32. Rubin MA, Mucci NR, Figurski J, Fecko A, Pienta KJ, Day ML. E-cadherin expression in prostate cancer: a broad survey using high-density tissue microarray technology. Hum Pathol. $2001 ; 32: 690-697$

33. Denu RA, Zasadil LM, Kanugh C, Laffin J, Weaver BA, Burkard ME. Centrosome amplification induces high grade features and is prognostic of worse outcomes in breast cancer. BMC Cancer. 2016;16:47.

34. Chatterjee SJ, McCaffrey L. Emerging role of cell polarity proteins in breast cancer progression and metastasis. Breast Cancer. 2014;6:15-27.

35. Ouyang X, Wang X, Xu K, Jin DY, Cheung AL, Tsao SW, et al. Effect of p53 on centrosome amplification in prostate cancer cells. Biochim Biophys Acta. 2001;1541:212-220.

36. Cosenza MR, Cazzola A, Rossberg A, Schieber NL, Konotop G, Bausch E, et al. Asymmetric centriole numbers at spindle poles cause chromosome missegregation in cancer. Cell Rep. 2017;20:1906-1920. 
38. Bello D, Webber MM, Kleinman HK, Wartinger DD, Rhim JS. Androgen responsive adult human prostatic epithelial cell lines immortalized by human papillomavirus 18. Carcinogenesis. 1997;18:1215-1223.

39. Cangemi R, Mensah A, Albertini V, Jain A, Mello-Grand M, Chiorino G, et al. Reduced expression and tumor suppressor function of the ETS transcription factor ESE-3 in prostate cancer. Oncogene. 2008;27:2877-2885.

40. Rickman DS, Soong TD, Moss B, Mosquera JM, Dlabal J, Terry S, et al. Oncogenemediated alterations in chromatin conformation. Proc Natl Acad Sci USA. 2012;109:90839088.

41. Shi Q, King RW. Chromosome nondisjunction yields tetraploid rather than aneuploid cells in human cell lines. Nature. 2005;437:1038-1042.

42. Bettencourt-Dias M, Rodrigues-Martins A, Carpenter L, Riparbelli M, Lehmann L, Gatt MK, et al. SAK/PLK4 is required for centriole duplication and flagella development. Curr Biol. 2005;15:2199-2207.

43. Habedanck R, Stierhof YD, Wilkinson CJ, Nigg EA. The Polo kinase Plk4 functions in centriole duplication. Nat Cell Biol. 2005;7:1140-1146.

44. Gregan J, Polakova S, Zhang L, Tolic-Norrelykke IM, Cimini D. Merotelic kinetochore 
attachment: causes and effects. Trends Cell Biol. 2011;21:374-381.

45. Wang M, Nagle RB, Knudsen BS, Rogers GC, Cress AE. A basal cell defect promotes budding of prostatic intraepithelial neoplasia. J Cell Sci. 2017;130:104-110.

46. Malhas AN, Vaux DJ. Nuclear envelope invaginations and cancer. Advances in experimental medicine and biology. 2014;773:523-535.

47. Xue JZ, Woo EM, Postow BT, Chait H, Funabiki H. Chromatin-bound Xenopus Dppa2 shapes the nucleus by locally inhibiting microtubule assembly. Dev. Cell. 2013;27:47-59.

48. Beheshti B, Park PC, Sweet JM, Trachtenberg J, Jewett MA, Squire JA. Evidence of chromosomal instability in prostate cancer determined by spectral karyotyping (SKY) and interphase fish analysis. Neoplasia. 2001;3:62-69.

49. Sun C, Dobi A, Mohamed A, Li H, Thangapazham RL, Furusato B, et al. TMPRSS2-ERG fusion, a common genomic alteration in prostate cancer activates C-MYC and abrogates prostate epithelial differentiation. Oncogene. 2008;27:5348-5353.

50. Zeng W, Sun H, Meng F, Liu Z, Xiong J, Zhou S, et al. Nuclear C-MYC expression level is associated with disease progression and potentially predictive of two year overall survival in prostate cancer. Int J Clin Exp Pathol. 2015;8:1878-1888. 
51. Lipponen P, Aaltomaa S, Tammi R, Tammi M, Agren U, Kosma VM. High stromal hyaluronan level is associated with poor differentiation and metastasis in prostate cancer. European journal of cancer. 2001;37:849-856.

52. Werner S, Pimenta-Marques A, Bettencourt-Dias M. Maintaining centrosomes and cilia. J Cell Sci. 2017;130:3789-3800.

53. Pimenta-Marques A, Bento I, Lopes CA, Duarte P, Jana SC, Bettencourt-Dias M. A mechanism for the elimination of the female gamete centrosome in Drosophila melanogaster. Science. 2016;353:aaf4866.

54. Pihan GA, Purohit A, Wallace J, Malhotra R, Liotta L, Doxsey SJ. Centrosome defects can account for cellular and genetic changes that characterize prostate cancer progression. Cancer Res. 2001;61:2212-2219.

55. Godinho SA, Picone R, Burute M, Dagher R, Su Y, Leung CT, et al. Oncogene-like induction of cellular invasion from centrosome amplification. Nature. 2014;510:167-171.

56. Kushner EJ, Ferro LS, Liu JY, Durrant JR, Rogers SL, Dudley AC, et al. Excess centrosomes disrupt endothelial cell migration via centrosome scattering. J. Cell Biol. 2014;206:257-272. 


\section{Figure Legends}

Fig. 1 Primary prostate tumors display centrosome loss.

a Normal human prostate tissue contains E-Cadherin (green) on cell borders and CEP135 (red)

on centrosomes. DNA (blue). Centrosomes in individual cells were identified within a complete z-series of the tissue. (Left) A single z-section shows normal gland structure. Scale, $100 \mu \mathrm{m}$. (Middle) Maximum intensity projection of region (white box in left panel) showing centrosome distribution. Scale, $10 \mu \mathrm{m}$. (Right) Selected single z-sections (from box in middle panel) displaying distinct centrosomes. Labels identify specific z-sections. Scale, $5 \mu \mathrm{m} ; 1 \mu \mathrm{m}$ (insets). b Centrosomes in a low grade (Gleason 3) PCa gland detected as in (a). Scale, $10 \mu \mathrm{m}$. Insets are from boxed regions in larger image and are maximum intensity projections showing cells with two (left) and zero centrosomes (right) at higher magnification. Scale, $5 \mu \mathrm{m}$.

c Region of high grade (Gleason 5) PCa. Centrosomes are absent in cancer cells (identified by positive E-Cadherin immunostaining; delineated by white-dashed lines) but are visible in the surrounding stroma (yellow arrowheads). Scale, $10 \mu \mathrm{m}$.

d, e Tissue of normal human mammary gland (d) and triple negative breast cancer (TNBC) (e). Scale, $100 \mu \mathrm{m}$ (left panels); $10 \mu \mathrm{m}$ (right panels); $5 \mu \mathrm{m}$ (large insets); $1 \mu \mathrm{m}$ (small insets). f Number of centrosomes (CEP135 foci or $\gamma$-Tubulin foci) per cell in normal prostate, PCa, normal breast, and TNBC tissue cells. $\mathrm{n}>100$ cells from 3 independent experiments in a total of 16 different patient samples. Data are means \pm SD. Cells with $>2$ centrosomes show amplification. Differences were tested for significance using unpaired t-tests; ${ }^{*} \mathrm{p}<0.05 ;{ }^{* *} \mathrm{p}<$ 0.01 .

Fig. 2 Centrosome numbers are altered in PCa lines. 
Immunostaining of centriole proteins CEP135 (red), CENT3 (green), or $\gamma$-tubulin (green) in primary (PEC) (a) and immortalized non-tumorigenic prostate epithelial cells (b-d), and PCa cell lines (e-i). DNA, blue. Boxed regions are shown at higher magnification in insets.

j PCa cell lines contain an altered centrosome number. Data are means $\pm \mathrm{SD}(\mathrm{n}=100$ cells, 2 independent experiments for PEC, and 3 independent experiments for all other cell lines). PCa lines displaying centriole loss (significantly larger \% of cells with 0 centrosomes) and amplification (significantly larger $\%$ with $>2$ centrosomes) are indicated by black and red lines, respectively. Significant differences were evaluated with a two-tailed, unpaired t-test; ${ }^{*} \mathrm{p}<0.05$; $* * \mathrm{p}<0.01 ; * * *, \mathrm{p}<0.001$

Fig. 3 Centrosome loss increases the frequency of mitotic errors, chromosome fragmentation and micronuclei.

a,b Percentages of RWPE1 or PrEC cells with the indicated centrosome number after treatment with centrinone ( $300 \mathrm{nM})$ for the indicated number of days, followed by washout (WO). (b) Centrinone treatment does not change centrosome number in RWPE1 cells expressing centrinone-resistant mutant Plk4(G95L). Data are means \pm SD ( $n=300$ cells, 3 independent experiments for each treatment or time point). Centrosome numbers in Plk4(G95L) expressing cells at Day 0 and Day10 of centrinone treatment are not significantly different. c Selected frames from time-lapse videos of representative mock and centrinone-treated mitotic RWPE1 and PrEC cells stably expressing histone H2B-GFP and CETN1-tdTomato (not shown) to label chromosomes and centrioles, respectively. Anaphase lagging chromosomes (red arrowheads) and small chromosome fragments (yellow arrowheads) are indicated. Numbers indicate time (mins) prior to anaphase onset. See Supplementary Videos 1-4. 
d Measurements of mitotic duration (nuclear envelope breakdown to telophase) in mock, DMSO, and centrinone-treated RWPE1 (top) and PrEC (bottom) cells. Centrinone treatment significantly slows mitotic progression. Number of cells analyzed: RWPE1: WT=87, DMSO=77, centrinone $=88 ;$ PrEC $: \mathrm{WT}=90, \mathrm{DMSO}=100$, centrinone $=100$. Data are means \pm SD. Significant differences tested using two-tailed, unpaired t-tests; $* * * *, p<0.0001$.

e Mitotic RWPE1 cell treated with centrinone and stained with Alexa Fluor ${ }^{\mathrm{TM}} 647$ Phalloidin (red) and expressing histone H2B-GFP (green). Arrowheads indicate chromosome fragments. Scale, $10 \mu \mathrm{m}$.

f Mitotic RWPE1 cell treated with centrinone and immunostained for centromeric protein CENP-C (red) and microtubules (green). DNA, blue. Arrowheads mark chromosome fragments lacking centromeric protein CENP-C. Scale, $10 \mu \mathrm{m}$. See Supplementary Videos 7 and 8. g Percentage of RWPE1, PWPE1-Plk4(G95L), PrEC, and PrEC-Plk4KO cells containing micronuclei. Data are means \pm SD ( $>300$ cells from 3 independent experiments). Statistical significance evaluated using a two-tailed, unpaired t-test; ${ }^{* *}, \mathrm{p}<0.01$; 'ns', not significant. h Centrinone-treated RWPE1 cells were immunostained for the centromeric protein CENP-C to establish the presence of centromeres within micronuclei. Data are means $\pm \operatorname{SD}(n>30$ micronuclei from 3 independent experiments).

i Spheroids of RWPE1 cells were treated with DMSO or centrinone from days 6 to 13, and stained on day 13 to label DNA. Arrowheads mark micronuclei. Scale, $10 \mu \mathrm{m}$.

Fig. 4 Centrosome loss produces abnormal nuclear envelopes and multinucleate cells whose number significantly increases with Gleason grade. 
a Selected frames from time-lapse movies of mock and centrinone-treated mitotic RWPE1H2B-GFP and PrEC-H2B-GFP cells. Arrowheads mark nuclear envelope defects including invaginated (red), multinucleated (green), and multilobed (orange) morphologies. Scale, $10 \mu \mathrm{m}$. b RWPE1 and PrEC cells were treated with DMSO or centrinone for 15 days and then stained with Alexa Fluor 647 Phalloidin (red) and nuclear envelope markers Lamin A/C or Emerin (green). DNA, blue. Arrowheads mark nuclear envelope invaginations. Scale, $10 \mu \mathrm{m}$. c,d Percentages of multinucleated cells after treatment with DMSO or centrinone for 15 days in RWPE1 and PrEC cells $(\mathbf{c})$ or in the indicated cell line $(\mathbf{d})$. Data are means \pm SD (n>300 cells from 3 independent experiments). Statistically analyzed using a two-tailed, unpaired t-test; ***, $\mathrm{p}<0.001 ; * * * *, \mathrm{p}<0.0001$.

e Human prostate cancer tissue contains multinucleated cells. Tissue was stained for E-Cadherin (green) and nuclear envelope markers Lamin-A/C or Emerin (red). DNA, blue. Dashed lines trace the E-Cadherin-positive cell borders of individual PCa cells. All images are single sections selected from a z-stack. Boxed regions are shown at higher magnification and highlight individual multinucleated cells. Arrowhead marks a micronucleus. Scale, $100 \mu \mathrm{m}$ in the upper left panel; $10 \mu \mathrm{m}$ in all other panels.

f Percentages of multinucleated cells of human PCa tissue. Data are means \pm SD. $n>50$ total cells from at least 3 different patients in a total 26 patient samples. Statistical differences tested using a two-tailed, unpaired t-test; *, p <0.05; **, p $<0.01$.

g Human prostate cancer tissue contains multinucleated cells that lack centrosomes. Tissue was immunostained for E-Cadherin (green) and CEP135 (red). DNA, blue. This maximum intensity projection shows a binucleate cell (yellow arrowhead), and dashed lines trace the two nuclei. Note the absence of CEP135 foci. Scale, $10 \mu \mathrm{m}$. 
h Most multinucleated cells in human PCa tissue have a decreased number ofcentrosomes $(<2$ CEP135 foci per cell). Data are means \pm SD. $n=18$ patients with multinucleated cells.

Statistical differences were tested using a two-tailed, unpaired t-test; *, $\mathrm{p}<0.05 ;{ }^{* *}, \mathrm{p}<0.01$.

Fig. 5 Centrosome loss results in aneuploidy and chromosomal rearrangements.

a Metaphase spreads of RWPE1 cells treated with DMSO or centrinone for 15 days. Arrowheads indicate chromosome fragments. Scale, $10 \mu \mathrm{m}$.

b Chromosome numbers per cell in mock, DMSO, or centrinone-treated RWPE1 cells. Data are means $\pm \mathrm{SD}$ ( $\mathrm{n}=40$ mock, $30 \mathrm{DMSO}$, and 79 centrinone-treated cells). Statistical differences were tested using a two-tailed, unpaired t-test with Welch's correction; ${ }^{* * *}, \mathrm{p}<0.001 ; * * * *, \mathrm{p}<$ 0.0001 .

c Cell cycle profiles of 15-day DMSO or centrinone treated RWPE1 and RWPE1-Plk4(G95L) cells analyzed by flow cytometry. Profiles are gated to show the percentages of cells with $2 \mathrm{~N}$ (diploid) and $>4 \mathrm{~N}$ (aneuploidy) DNA content. Note that the frequency of aneuploidy increases $>6$-fold after centrinone treatment but not in centrinone-resistant Plk4 (G95L) mutant RWPE1 cells.

d, e Representative spectral karyotyping (SKY) images of passage 9 RWPE1 cells grown in keratinocyte serum-free medium (d) and passage 18 RWPE1 cells grown with 10\% FBS (e). Cells were mock or centrinone treated for 15 days.

f, g Heat-maps of chromosome copy numbers determined by SKY from RWPE1 cells in (d) and (e). Twenty cells were analyzed for each condition. Heat scales indicate chromosome copy number. "X", no detection. 
Fig. 6 Chronic or transient centrosome loss in non-tumorigenic cells increases their potential to form malignant xenograft tumors.

a Number of xenograft tumors formed from parental PrEC (4 injections), PrEC-Plk4KO-1 (8 injections), and PrEC-Plk4KO-2 (8 injections) cell lines.

b, c Sections of PrEC-Plk4KO tumor stained with hematoxylin and eosin. (b) Low magnification of tumor section shows streams of tumor cells invading surrounding smooth muscle and fat layers. (c) Tumor section at low (left) and high (right) magnification shows proliferative cells and nuclear atypia. Mitotic cells (red arrowhead), enlarged nuclei (green arrowhead), and multinucleated cells (yellow arrowheads) are indicated. Scale, $100 \mu \mathrm{m}$.

d Scheme used to select PrEC clones following transient centrosome depletion. PrEC cells were treated with centrinone $(300 \mathrm{nM})$ for 15 days and cultured in normal growth medium after monoclonal expansion. Clones containing MYC and ERG protein levels consistent with PCa progression were selected (CTN1, 6, and 9) and subcutaneously injected into NOD/SCID male mice.

e Tumor volume growth curves from CTN-1, 6, and 9 injections (12 injections for each CTN clone are plotted). Number of tumors formed is indicated above.

f Kaplan-Meier tumor-free survival as evaluated by the presence of xenograft tumors. Data represent $n=12$ injection sites per clone $(\mathrm{CTN}-1,6$, and 9), total of 9 mice.

g Centrinone-derived PrEC tumor stained with hematoxylin and eosin (H\&E). Boxed region is shown at higher magnification (right). Scale, $100 \mu \mathrm{m}$.

h An H\&E-stained tissue section of a centrinone-derived PrEC tumor showing invasion through the adjacent muscle layer. Arrowheads indicate strands of tumor cells invading muscle. Scale, $100 \mu \mathrm{m}$. 
i CTN-9 tumor section immunostained for centrosome marker CEP135 (red). DNA, blue. Individual cells are shown at higher magnification in the boxed regions. Scale, $10 \mu \mathrm{m} ; 1 \mu \mathrm{m}$ right panels.

j Mitotic cells in CTN-9 tumors containing centrosome-associated (left) and acentrosomal (right) spindles. CEP135 (red), microtubules (green) and DNA (blue). Scale, $10 \mu \mathrm{m}$.

k Centrosome numbers in CTN tumors. $n=100$ number of cells counted per tumor. 


\section{Supplementary Figure Legends}

Fig. S1 Centrosome markers co-localize in human prostate cancer FFPE tissue samples. Human prostate cancer FFPE tissue sample immunostained for a pericentriolar material marker $\gamma$-tubulin (green), an epithelial cell border marker CD49f/integrin $\alpha 6$ (red, left panel), or the centriole marker CEP135 (red, middle panel). Hoechst-stained DNA, blue. Left panel scale, 100 $\mu \mathrm{m}$. Boxed region in left panel is shown at higher magnification in middle panel. Middle panel scale, $10 \mu \mathrm{m}$. Arrowhead marks two CEP135 foci in an individual cell shown at higher magnification in the right panels. Right panel scale, $0.125 \mu \mathrm{m}$.

Fig. S2 Centrosome loss decreases the growth rate in immortalized prostate epithelial RWPE1 cells and prolongs mitosis in both RWPE1 and PrEC cells.

a Cross-correlative scatter plots show cell cycle profiles of RWPE1 cells treated with DMSO or centrinone. Cells were stained with 7-AAD to label DNA and immunostained with antiphospho-histone $\mathrm{H} 3$ antibody (pH3) to label mitotic cells, and then analyzed by flow cytometry. b Quantification of the mitotic indices of RWPE1 cells and RWPE1-Plk4(G95L) cells treated with DMSO or centrinone. Cells were labeled with the DNA stain, 7-AAD, immunostained with mitosis-specific anti-phospho-histone $\mathrm{H} 3$ antibody (pH3), and analyzed by flow cytometry. Data are means $\pm \mathrm{SD}$ (3 independent experiments). Differences were tested for statistical significance using a two-tailed, unpaired t-test; ${ }^{* * * *}, \mathrm{p}<0.0001 ;$ ' $\mathrm{ns}$ ', not significant.

c Images showing nuclei and micronuclei (yellow arrowheads) in Hoechst-stained RWPE1 cells treated with DMSO or centrinone. Scale, $10 \mu \mathrm{m}$.

d Agarose gel of products of PCR reactions to detect Plk4 DNA. The presence of Plk4encoding genomic DNA in control (wild-type Plk4; WT) PrEC cell lines and CRISPR-generated 
Plk4-knock-out (Plk4KO) PrEC clones was tested by PCR, using purified genomic DNA as templates and PCR primers flanking the CRISPR guide-cutting region.

e q-RT-PCR analysis of the PrEC control and PrEC-Plk4KO clonal cells. Plk4 mRNA is absent in the Plk4KO clones. Data (ratios of measured Plk4:GAPDH) are normalized to the ratio of the parental (WT) PrEC cells.

Fig. S3 Chromosomal rearrangements in centrinone-treated RWPE1 and PrEC cells.

a, b SKY analysis of centrinone-treated RWPE1 cells (a) and PrEC CTN-9 cells (b) revealed chromosome translocations unique to treated cells that were not seen in untreated cells (though some translocations were seen in untreated cells [Fig. S5a]). Numbers indicate the source chromosomes for the fragments present in the translocation products.

Fig. S4 Screening centrinone-treated PrEC clones for PCa features.

a, b C-MYC (a) or ERG (b) protein levels in clonal cell populations were measured by flow cytometry. Protein levels were measured in untreated PrEC cells as well as 10 different PrEC clonal lines derived after DMSO or centrinone treatment. The fold change of C-MYC or EGR protein level relative to the level in untreated PrEC cells is plotted for each cell line. Clones 1,6, and 9 were selected for injection and evaluated for tumor formation in mice.

c Graph shows mean percentages of cells with the indicated number of centrosomes. Data are shown as means $\pm \mathrm{SD}$ ( $\mathrm{n}=300$ cells from 3 independent experiments). No significant differences were noted between these cell lines. Centrosomes were counted after cells recovered from treatments (explaining the restoration of normal centrosome numbers in centrinone-treated cells) and just prior to injection of the cells into mice. 
d The percentages of cells containing micronuclei. Data are means $\pm \operatorname{SD}(\mathrm{n}=300$ from 3 independent experiments). Differences were tested for statistical significance using a two-tailed, unpaired t-test; ${ }^{*}, \mathrm{p}<0.05 ;{ }^{* * *}, \mathrm{p}<0.001 ;{ }^{* * * *}, \mathrm{p}<0.0001$.

d Images of mitotic chromosome spreads prepared from the untreated PrEC (initial) cell line and the centrinone-treated clonal lines, before injection. The number of chromosomes in each spread is noted (top right corner).

Fig. S5 Characterization of PrEC-CTN xenograft tumors and cells isolated from CTN tumors. a, b Representative SKY images of an untreated PrEC cell (a) and a PrEC clone 9 (CTN-9) cell (b). Arrowheads indicate examples of chromosomal rearrangements.

c Expression levels of different epithelial and mesenchymal markers expressed in CTN-9 tumors. Protein levels were determined from the immunostaining intensities of the FFPE tissue section. Mucin production was detected by muscicarmine staining. -/-, not detectable; +/heterogeneously expressed; ++, strongly expressed.

d Immunofluorescence images of cells from a CTN-9 xenograft tumor (top row) and from human PCa tissue (bottom row) immunostained with the indicated epithelial, mesenchymal, and secretory markers. The human PCa tissue served as a positive control for immunostaining. Scale, $10 \mu \mathrm{m}$. 


\section{Supplementary Video Legends}

Supplementary Video 1. Mitosis in a mock-treated RWPE1 cell.

Time-lapse movie shows a representative mitotic RWPE1 cell stably co-expressing histone H2B-GFP (green) and CNTN1-tdTomato (red) to label chromosomes and centrioles, respectively. This cell established a bipolar spindle and segregated its duplicated genome into two daughter cells. Frames were generated from maximum intensity projections of z-stacks captured every 5 minutes. Scale, $10 \mu \mathrm{m}$.

Supplementary Video 2. Mitosis in a centrinone-treated RWPE1 cell.

Time-lapse movie of a representative centrinone-treated mitotic PrEC cell stably coexpressing histone H2B-GFP and CETN1-tdTomato. This centrosome-less cell displayed a prolonged delay in prometaphase with a central clustered chromosome mass. Eventually, the cell displays cytokinesis failure, generating a single multinucleated daughter cell. Each frame is a maximum intensity projection of a z-stack captured every 5 minutes. Scale, $10 \mu \mathrm{m}$.

Supplementary Video 3. Mitosis in a mock-treated RWPE1 cell.

Time-lapse movie shows a representative mitotic RWPE1 cell stably expressing histone H2BGFP. This cell established a bipolar spindle and segregated its duplicated genome into two daughter cells. Frames were generated from maximum intensity projections of z-stacks captured every 5 minutes. Scale, $10 \mu \mathrm{m}$.

Supplementary Video 4. Mitosis in a mock-treated PrEC cell.

Time-lapse movie of a representative mitotic PrEC cell stably expressing histone H2B-GFP. 
This cell established a bipolar spindle and segregated its duplicated genome into two daughter cells. Each frame is a maximum intensity projection of a z-stack captured every 5 minutes. Scale, $10 \mu \mathrm{m}$.

Supplementary Video 5. Mitosis in a centrinone-treated RWPE1 cell.

Time-lapse movie of a representative centrinone-treated mitotic RWPE1 cell stably expressing histone H2B-GFP. This cell established a bipolar spindle and segregated its duplicated genome. Note the prolonged delay in prometaphase and monopolar spindle. Subsequently, the cell displays cytokinesis failure, generating a single multinucleated daughter cell. Chromosome fragments are present throughout mitosis. Each frame is a maximum intensity projection of a z-stack captured every 6 minutes. Scale, $10 \mu \mathrm{m}$.

Supplementary Video 6. Mitosis in a centrinone-treated PrEC cell.

Time-lapse movie of a representative centrinone-treated mitotic PrEC cell stably expressing histone H2B-GFP. At the beginning of the movie, this cell contains a monopolar spindle with peripheral small H2B-containing elements, which are presumably unattached chromosome fragments. Eventually the spindle becomes pseudo-bipolar. Lagging chromosomes and chromosome fragments are visible during anaphase. Each frame is a maximum intensity projection of a z-stack captured every 5 minutes. Scale, $10 \mu \mathrm{m}$.

Supplementary Video 7. Chromosome fragments in a centrinone-treated RWPE1 mitotic cell. Movie generated by stepping through each section of a z-stack taken of a centrinone-treated mitotic RWPE1 cell. The cell was immunostained for $\alpha$-tubulin (green) to mark microtubules 
and CENP-C (red) to mark centromeres. DNA (blue). Note the presence of small chromosome fragments lacking CENP-C and not attached to microtubules. The step size for each z-section was $0.2 \mathrm{um}$, for a total stack depth of $7.8 \mathrm{um}$. Scale, $10 \mu \mathrm{m}$.

Supplementary Video 8. Bipolar spindle in a centrinone-treated RWPE1 mitotic cell. Movie generated by stepping through each section of a z-stack taken of a centrinone-treated mitotic RWPE1 cell immunostained for $\alpha$-tubulin (green) to mark microtubules, and CENP-C (red) to mark centromeres. DNA (blue). Note the acentrosomal, bipolar spindle. The step size for each z-section was $0.2 \mathrm{um}$, for a total stack depth of $7.8 \mathrm{um}$. Scale, $10 \mu \mathrm{m}$.

Supplementary Video 9. Abnormal nuclear envelope reformation in a dividing centrinonetreated RWPE1 cell.

Time-lapse movie of a centrinone-treated mitotic RWPE1 cell stably expressing histone H2BGFP. The cell eventually establishes a bipolar spindle and proceeds through anaphase A with no obvious defects. However, chromosomes reform into abnormally-shaped nuclei during telophase. Each frame is a maximum intensity projection of a z-stack captured every 5 minutes. Scale, $10 \mu \mathrm{m}$.

Supplementary Video 10. Abnormal nuclear envelope reformation in a dividing centrinonetreated PrEC cell.

Time-lapse movie of a centrinone-treated mitotic PrEC cell stably expressing histone H2BGFP. A bipolar spindle eventually formed, and anaphase A proceeded with no obvious defects. However, abnormally-shaped nuclear envelopes reformed during telophase. Each 
frame is a maximum intensity projection of a z-stack captured every 5 minutes. Scale, $10 \mu \mathrm{m}$. 


\section{Figure 1}

A

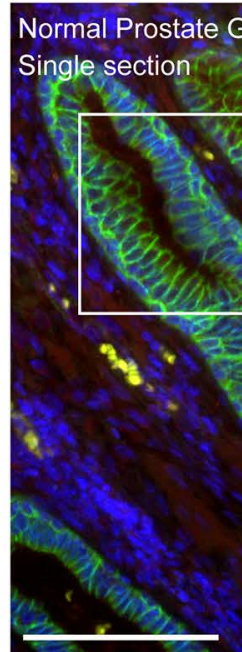

B E-Cadherin CEP135

Prostate Cancer leason 3

Projection

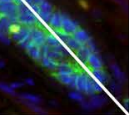

Luminal cells

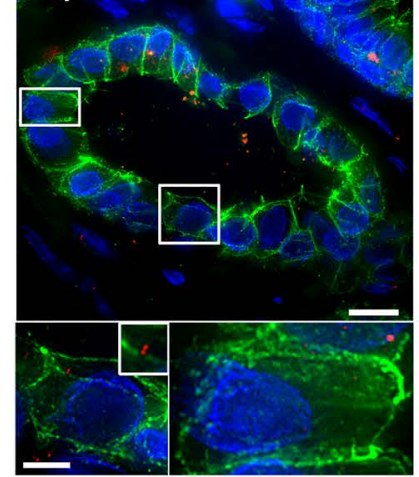

C

E-Cadherin CEP135 Prostate Cancer Gleason 5 Projection

D

E-Cadherin CEP135
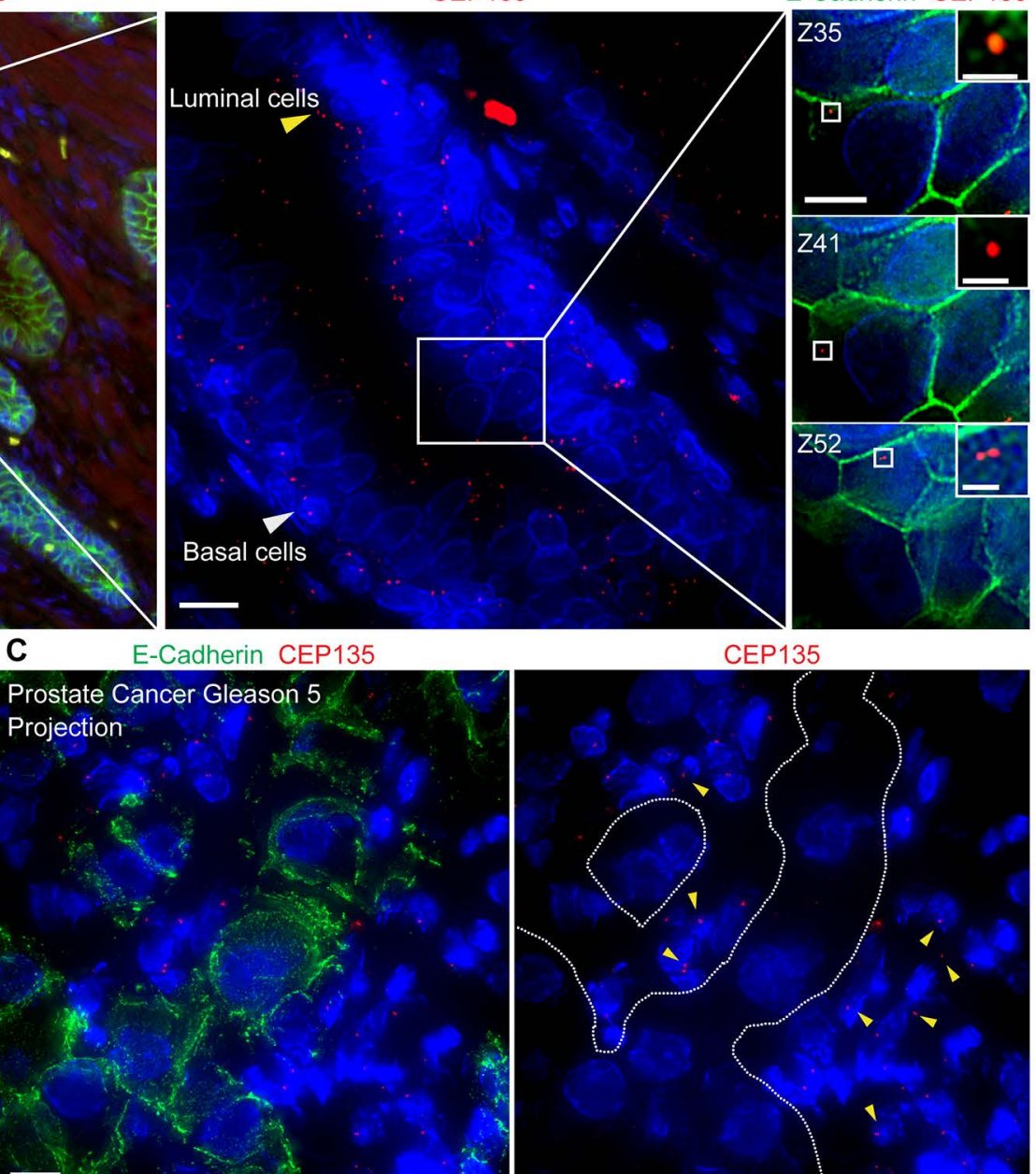

Basal cells

CEP135

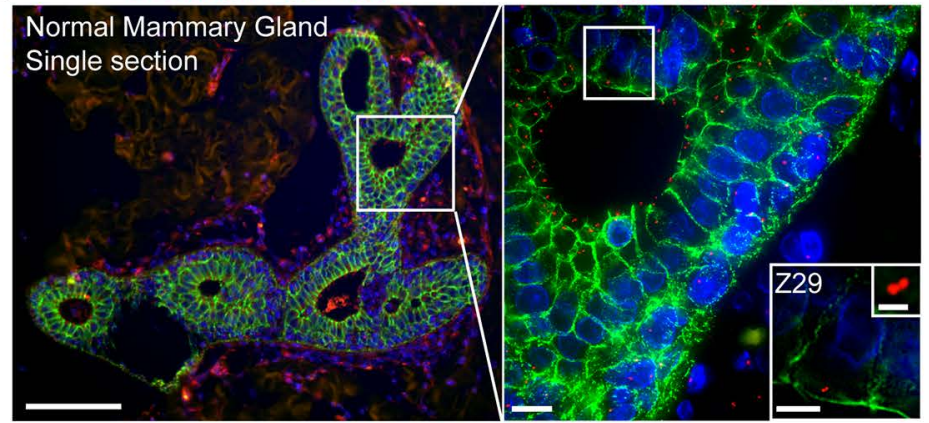

E-Cadherin CEP135

E E-Cadherin CEP135 E-Cadherin CEP135

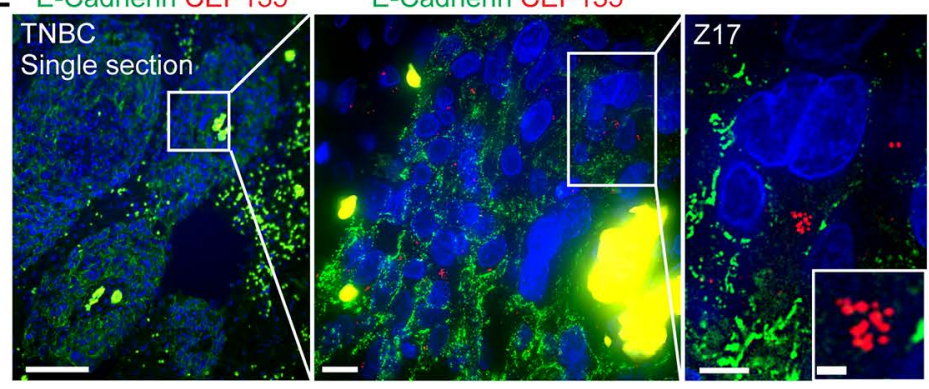

$\mathbf{F}$
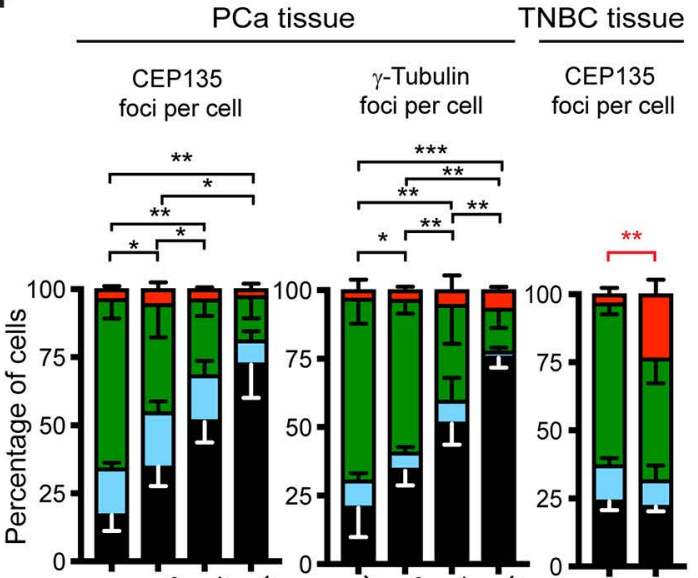

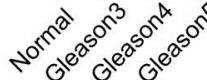
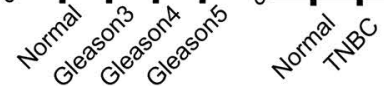

$\square 0 \square 1 \square 2 \square>2$ foci 
Figure 3

A

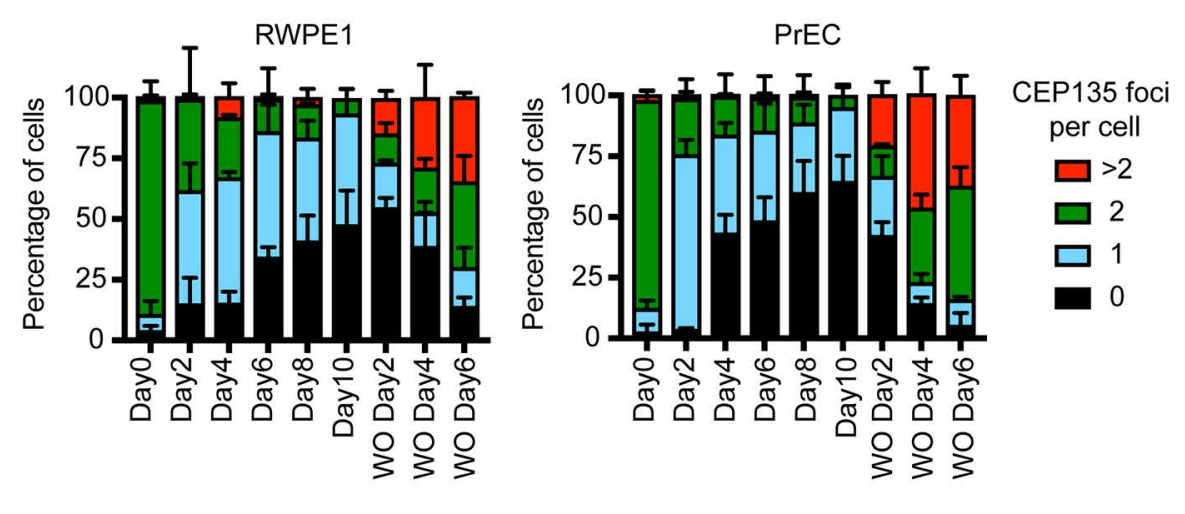

B

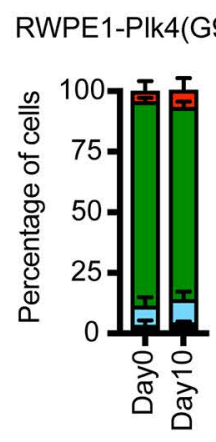

D

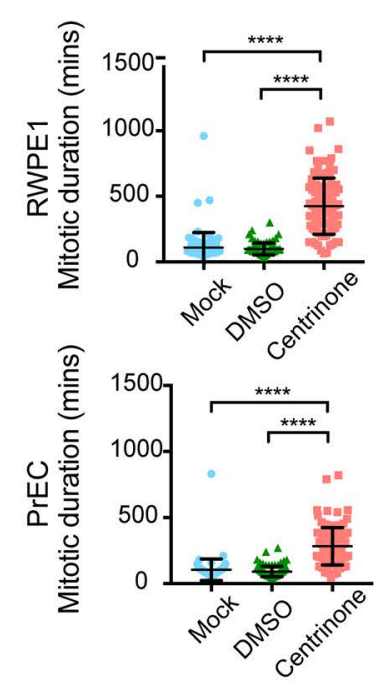

E H2B-GFP Phalloidin

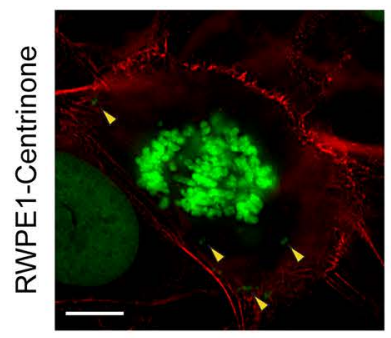

H

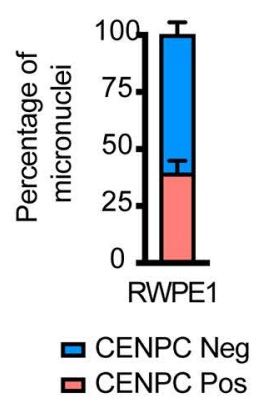

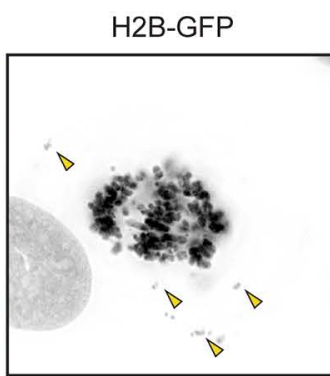

RWPE1 RWPE1-Plk4(G95L)

PrEC

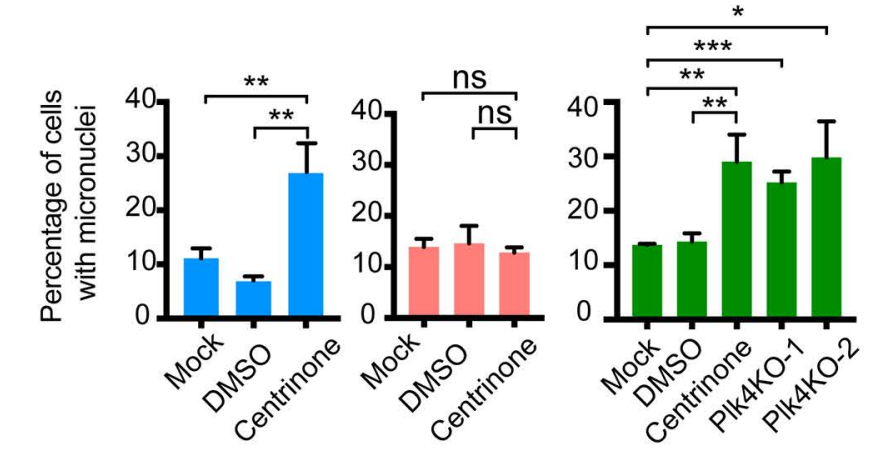

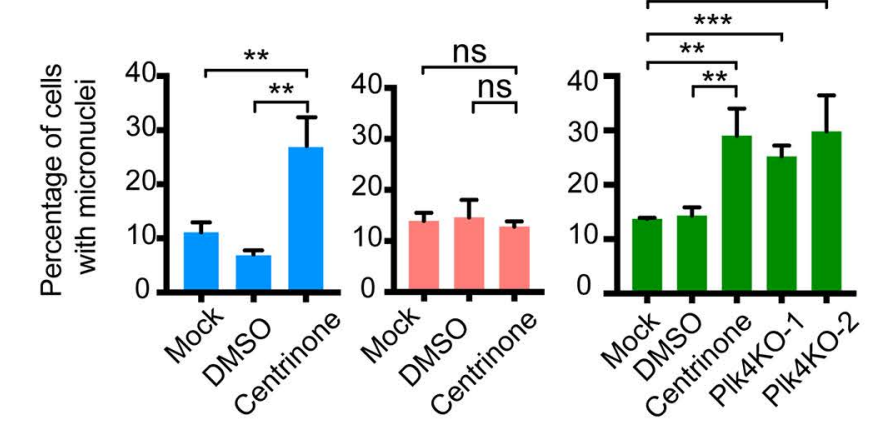

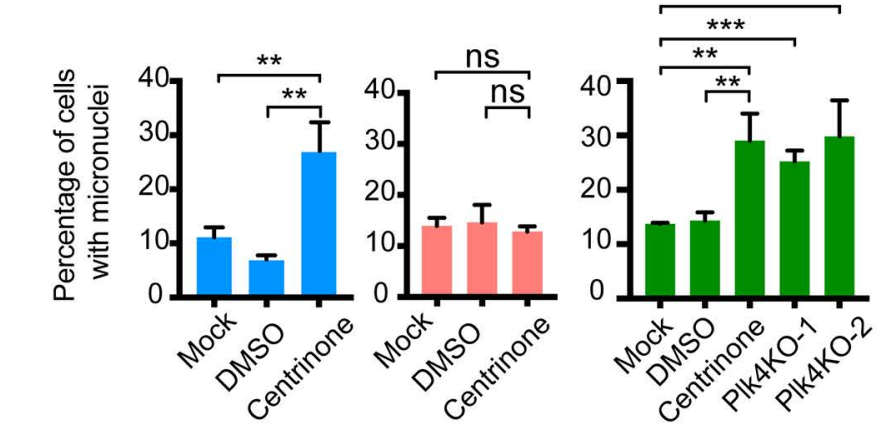

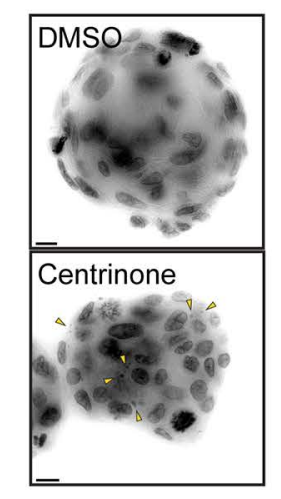

G

CENP-C

号

$+\frac{1}{2 x}$

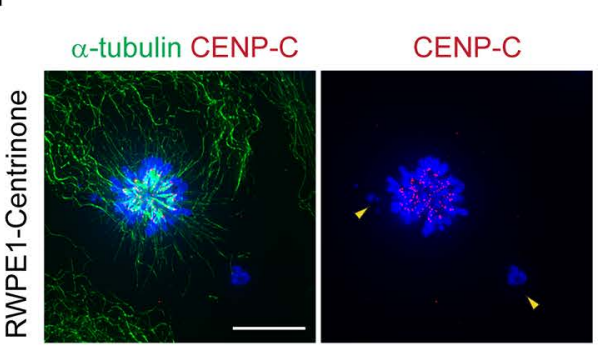




\section{Figure 5 \\ A}
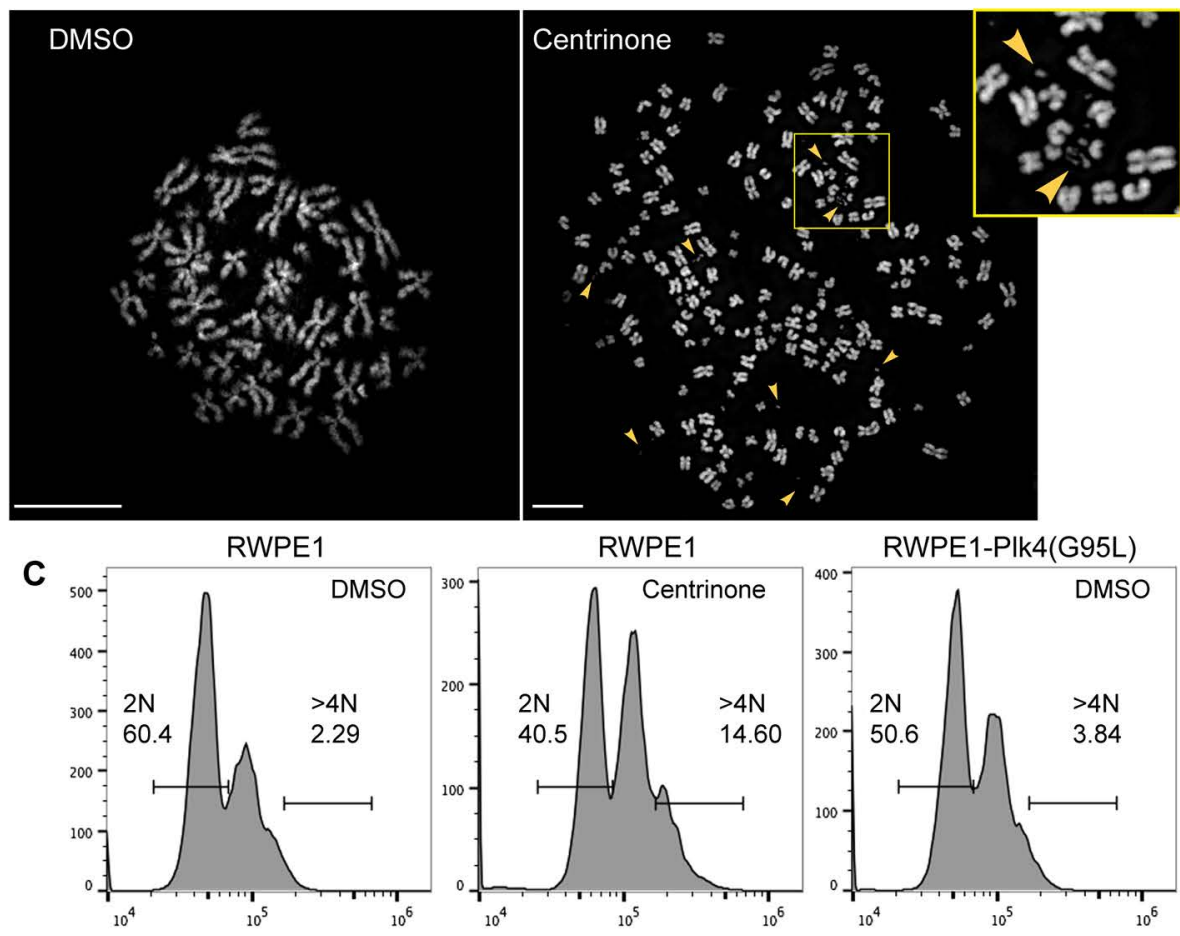

Serum free-Mock

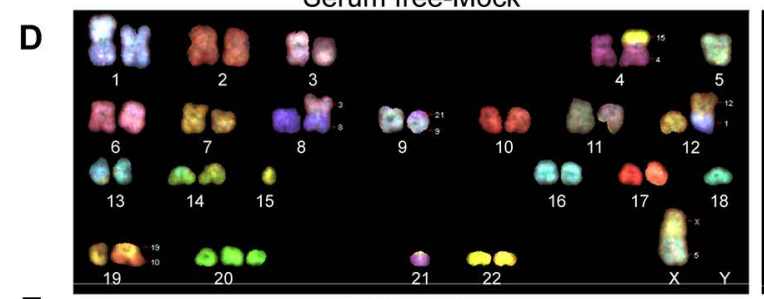

E

FBS-Mock

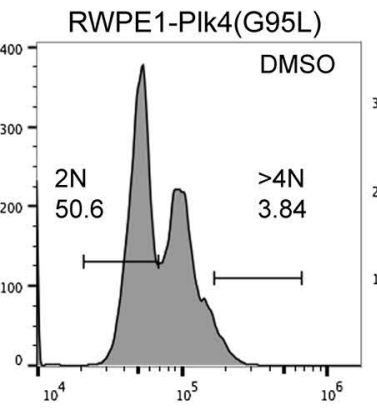

Serum free-Centrinone
B

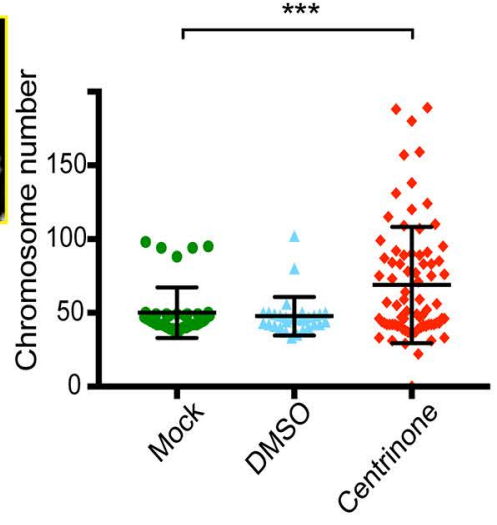

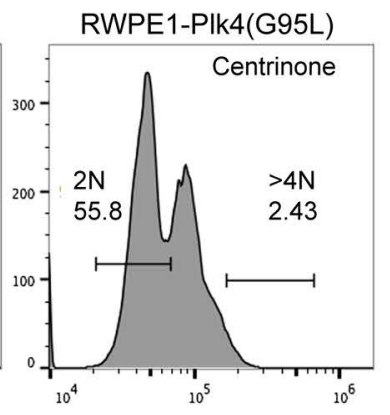

RWPE1-PIk4(G95L)

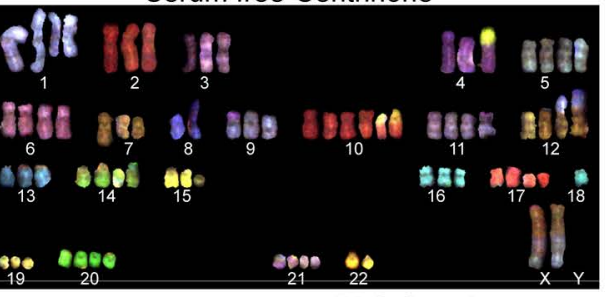

FBS-Centrinone

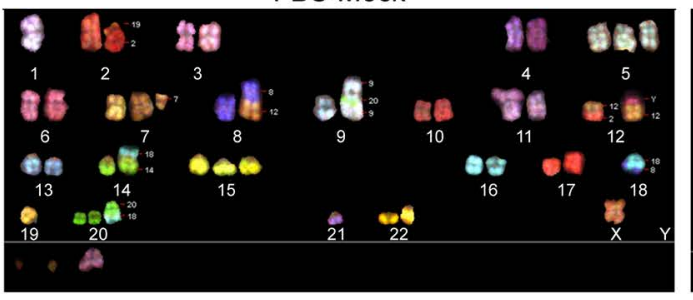

$\mathbf{F}$

Chromosome

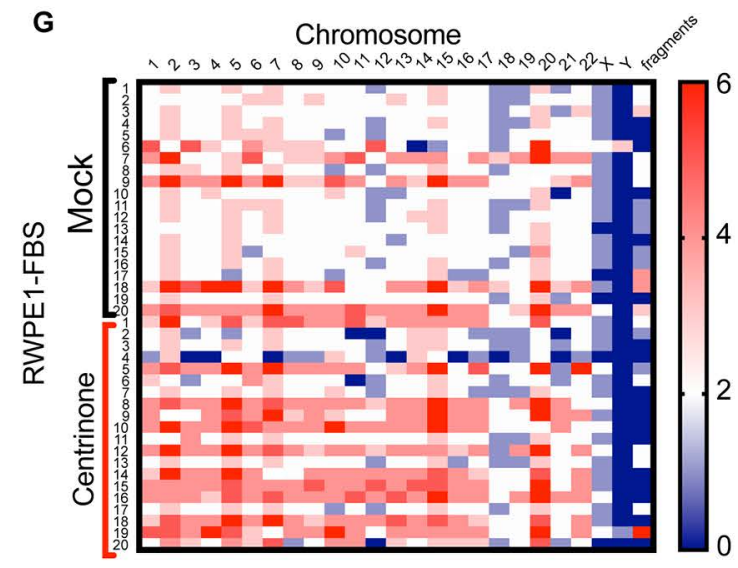

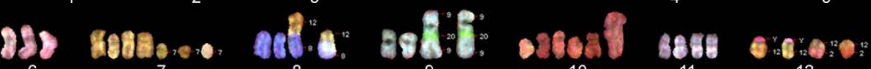

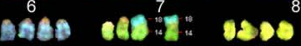

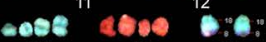

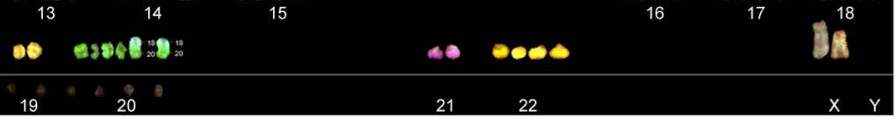


Figure 6

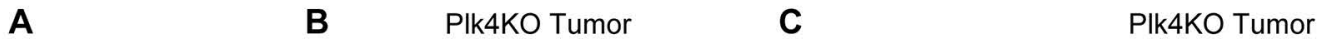

\begin{tabular}{|c|c|}
\hline Cell line & Tumors \\
\hline PrEC & $0 / 4$ \\
\hline Plk4KO-1 & $2 / 8$ \\
\hline Plk4KO-2 & $2 / 8$ \\
\hline
\end{tabular}
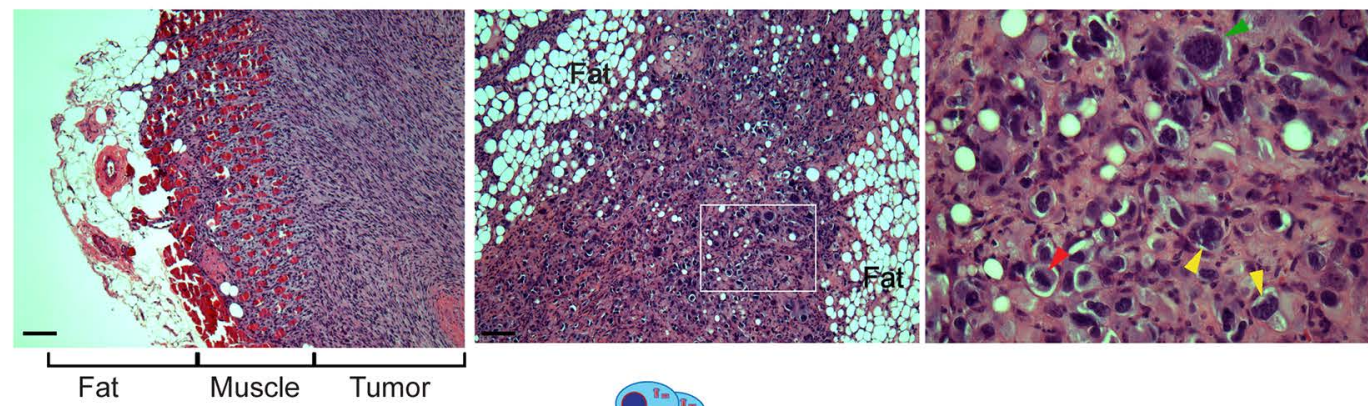

D PrEC cells
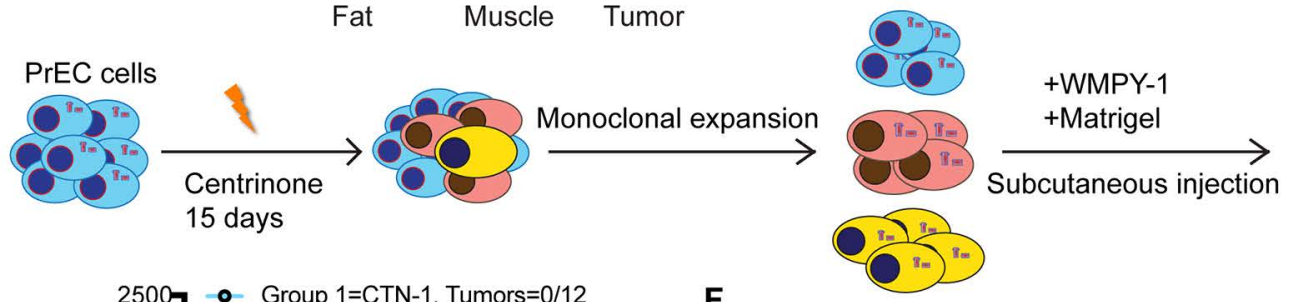

E

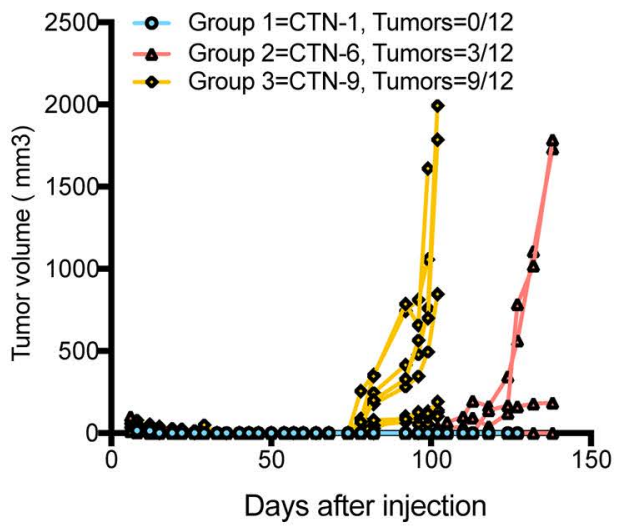

F

CTN Tumor

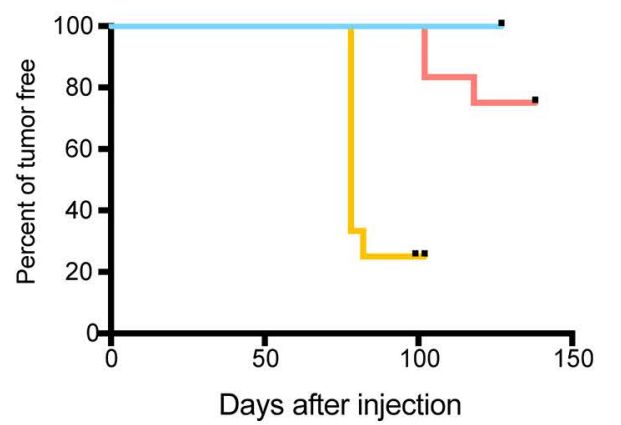

G

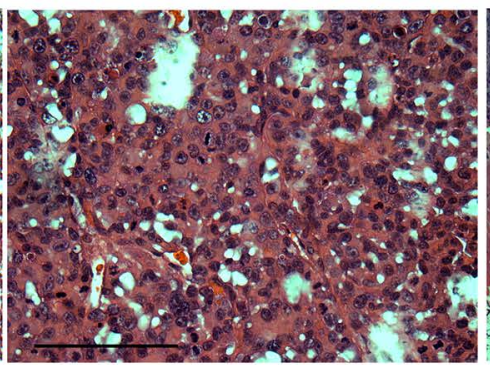

H

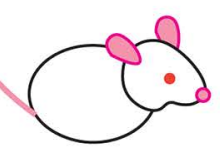

- CTN-1

- CTN-6

- CTN-9
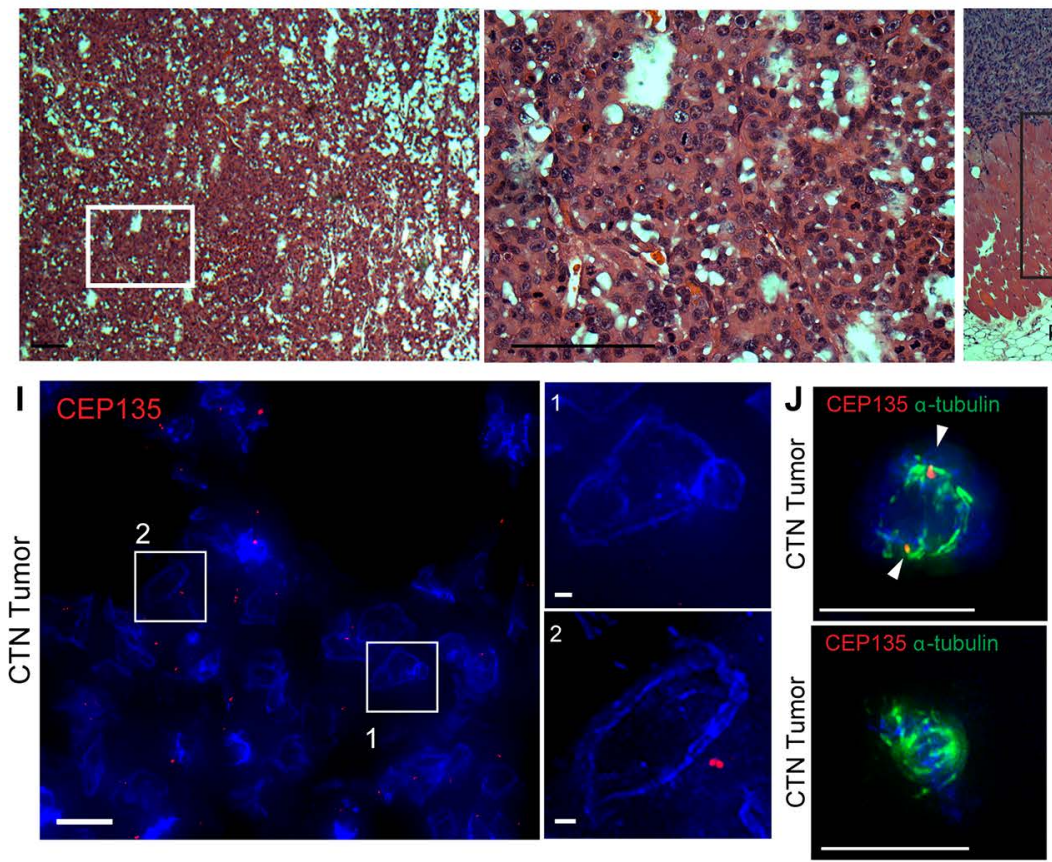

CTN Tumor
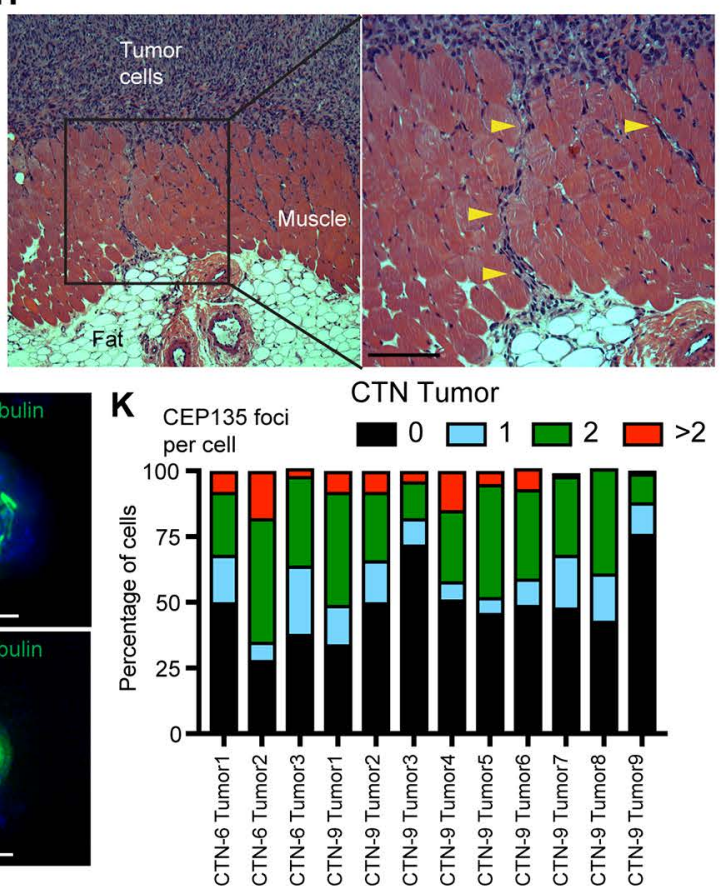
Figure S1

CD49f ( $\alpha 6$ integrin) $\gamma$-Tubulin

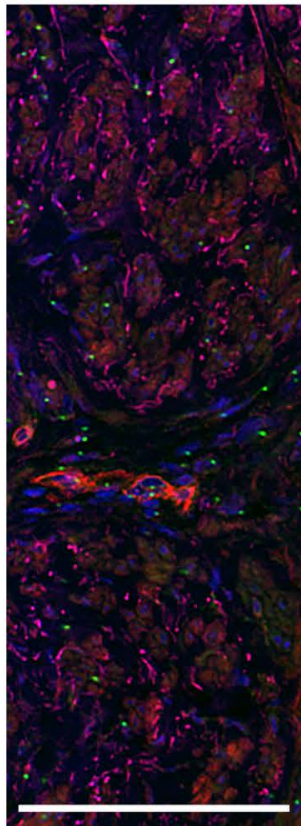

CEP135 $\gamma$-Tubulin

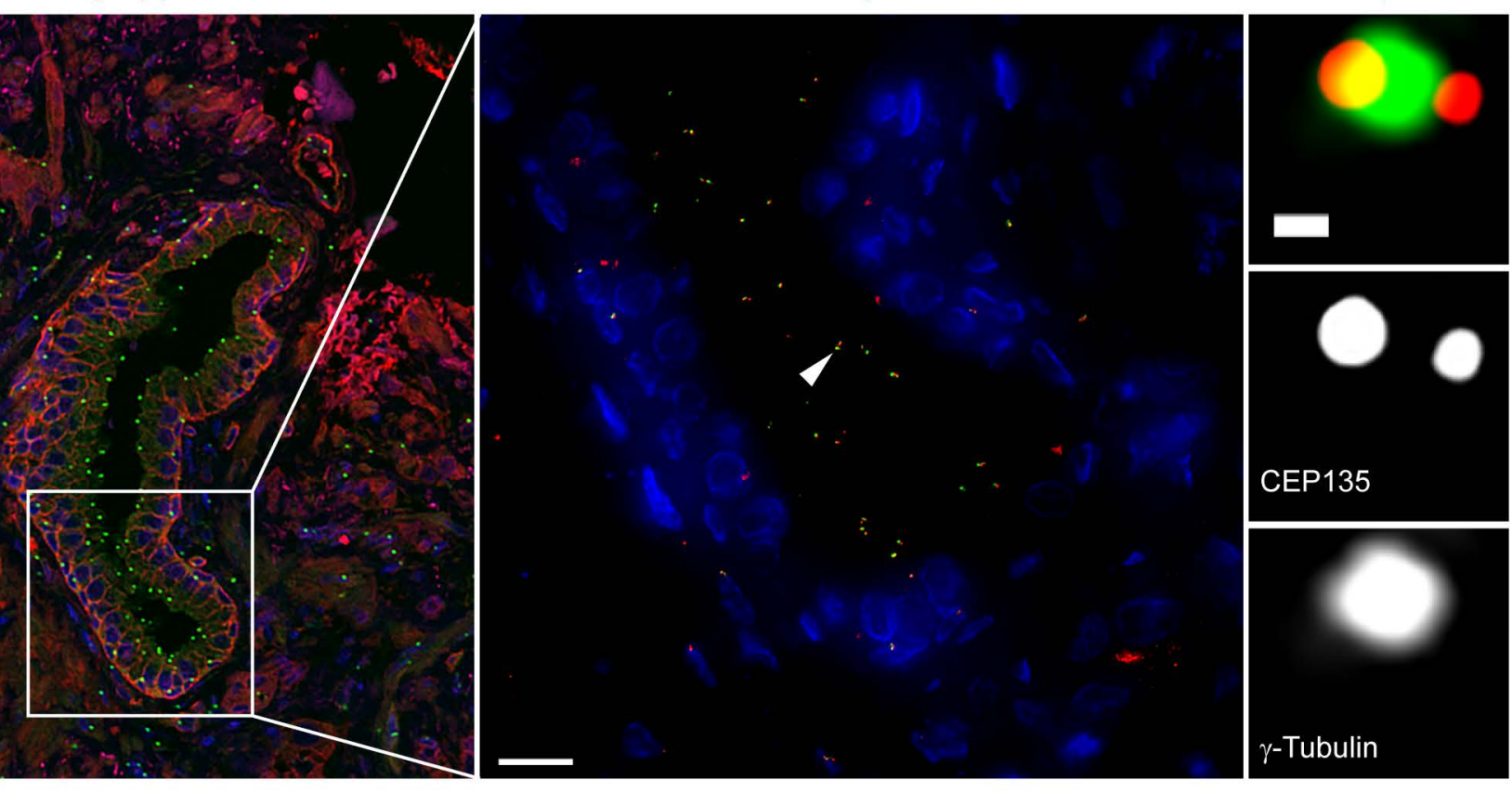


Figure S2

A

RWPE1

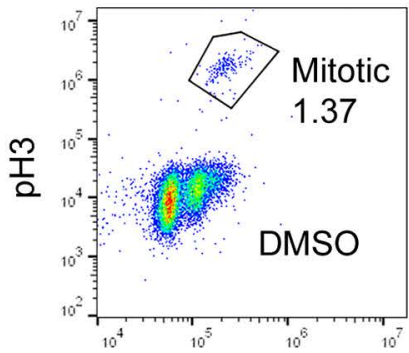

7AAD
B

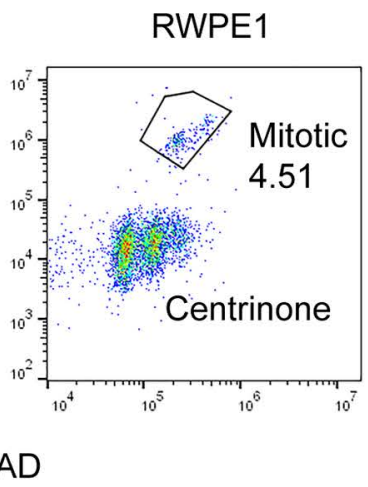

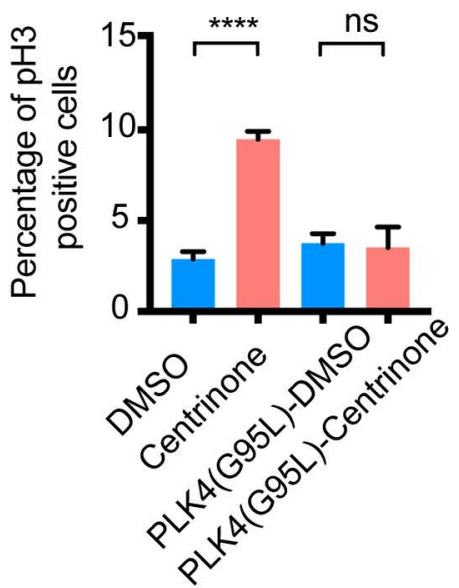

C

RWPE1

DMSO
RWPE1

Centrinone
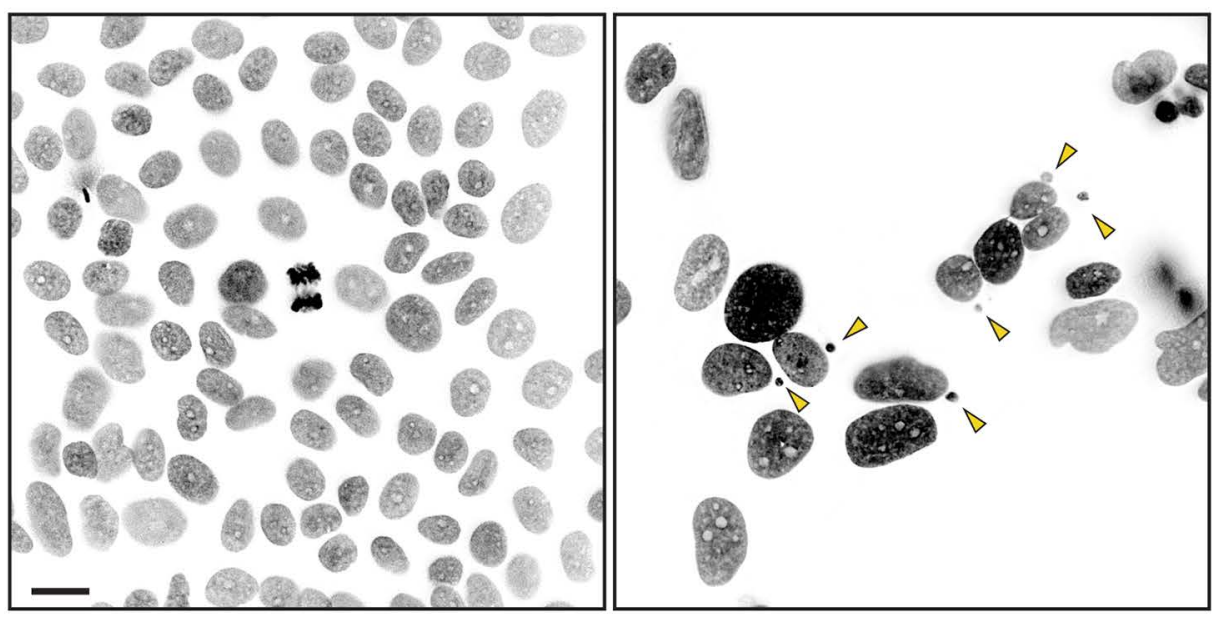

D

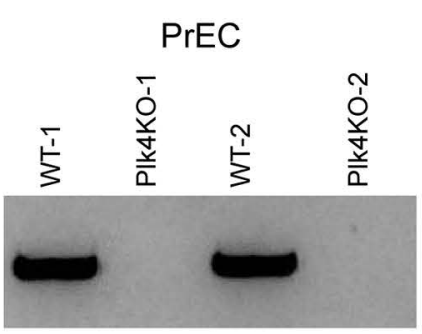

E

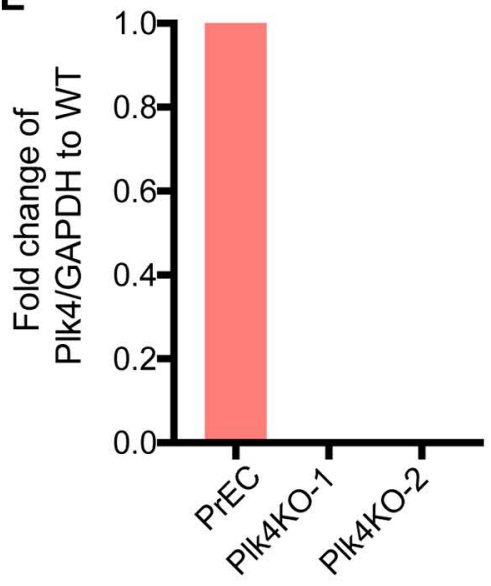


Figure S3

A

B

RWPE1 Centrinone
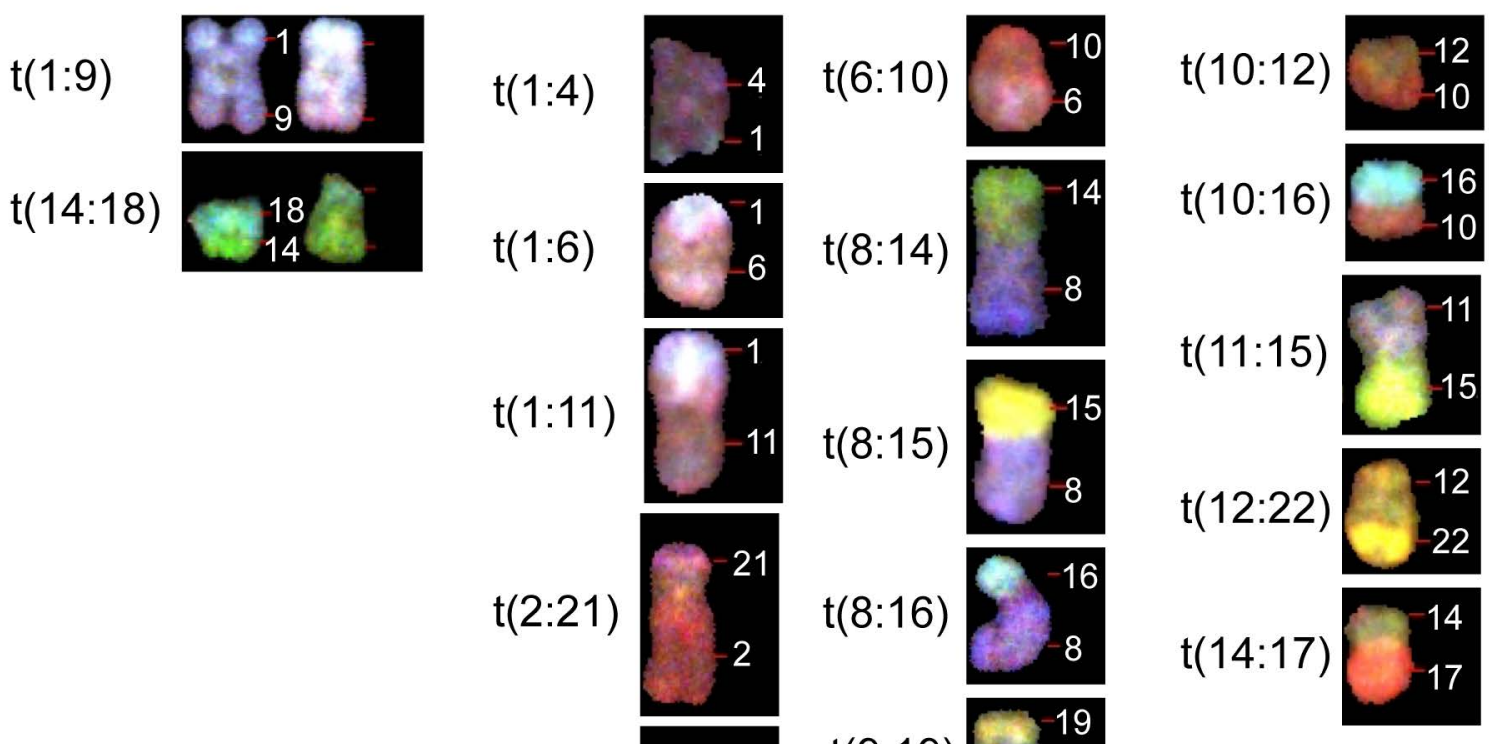

$t(4: 8)$

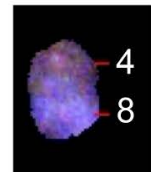

$t(9: 19)$ 


\section{Figure S4}

A

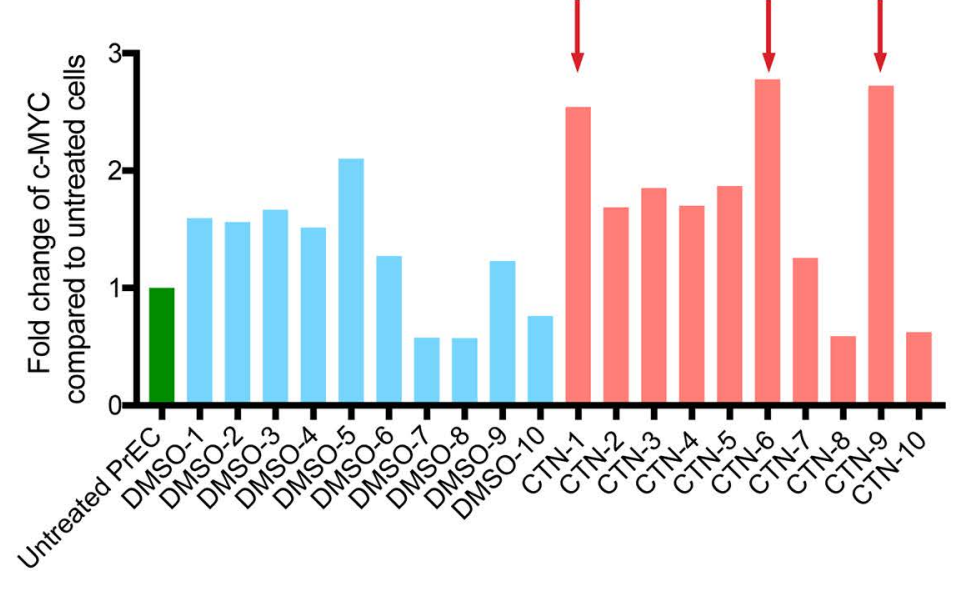

B

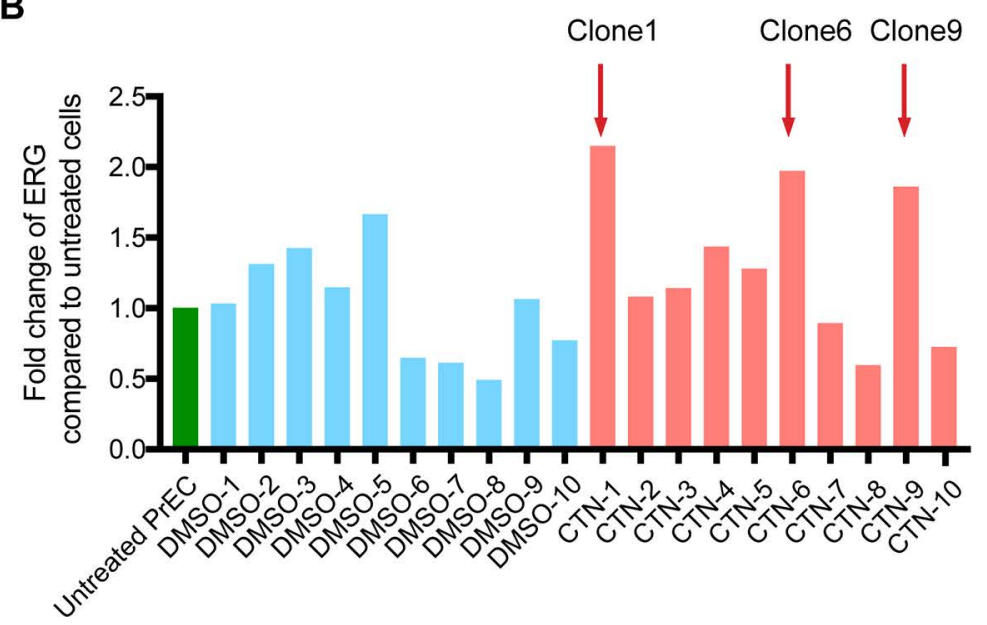

C

Centrosome numbers per cell

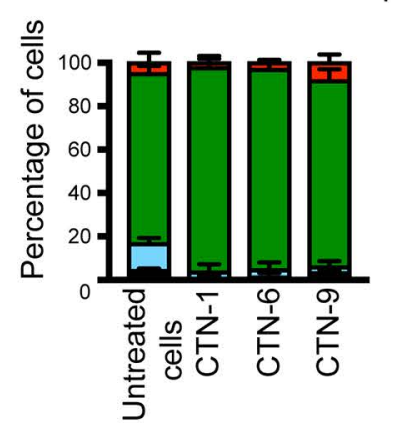

CEP135 foci per cell

므

며 2
미 0

E

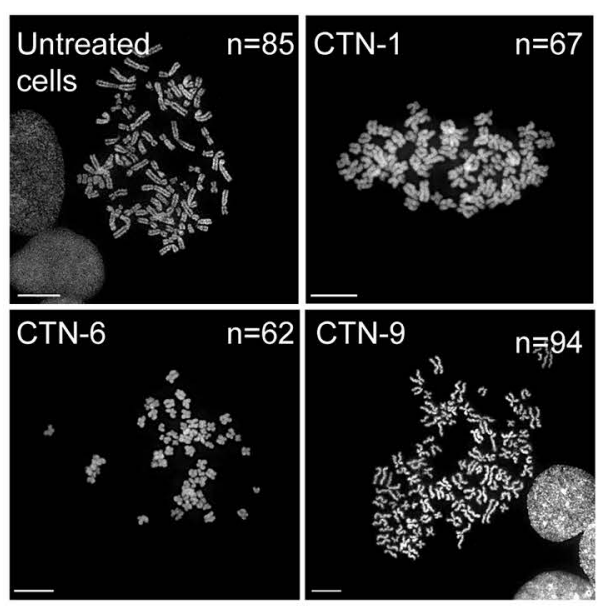

Micronuclei per cell

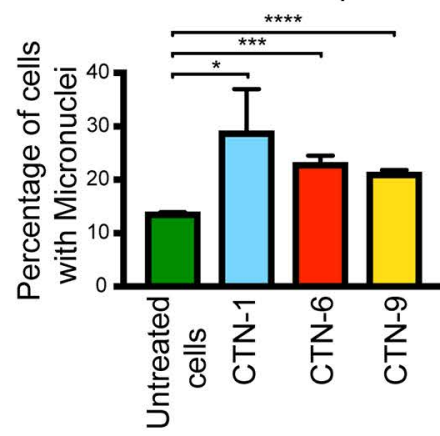




\section{Figure S5}

A

Untreated PrEC

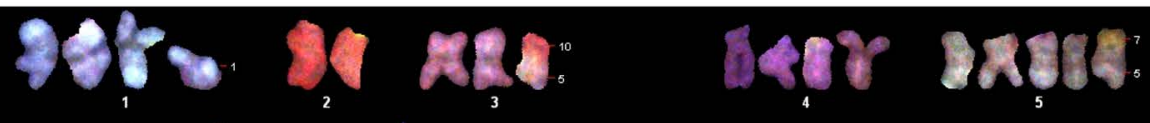

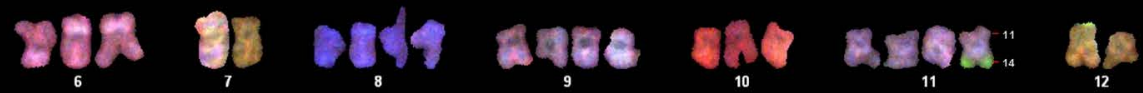

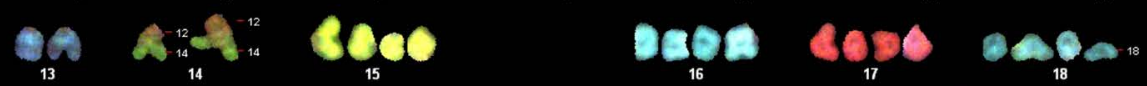

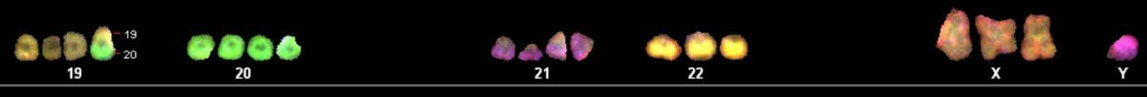

B

PrEC CTN-9

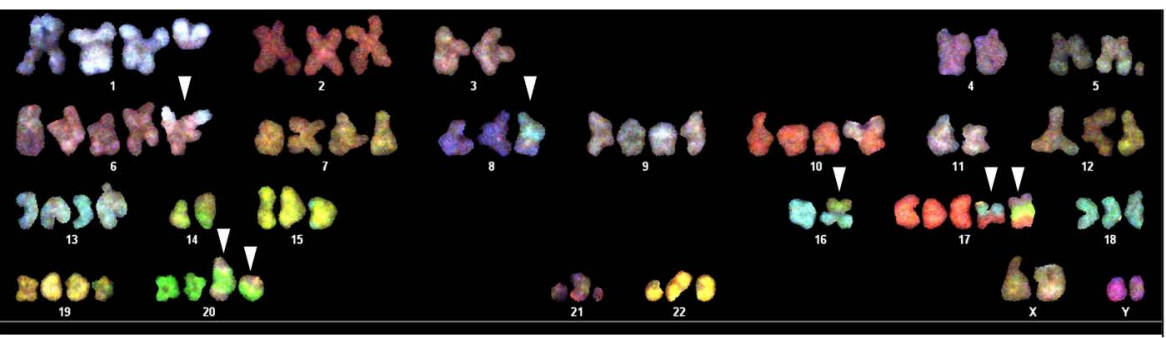

C

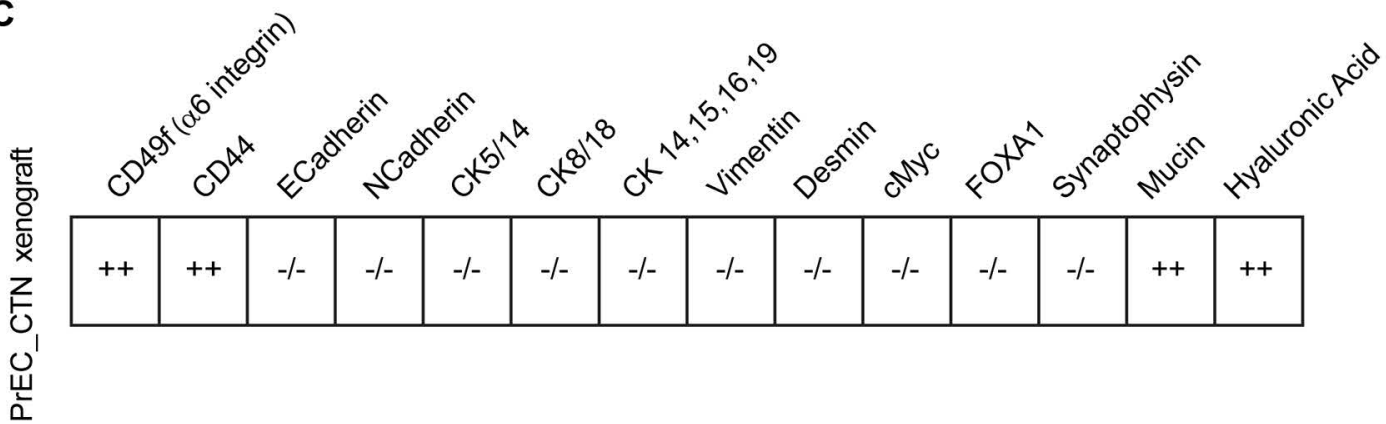

D

CD44 HA

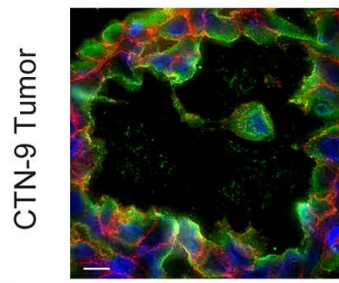

믐

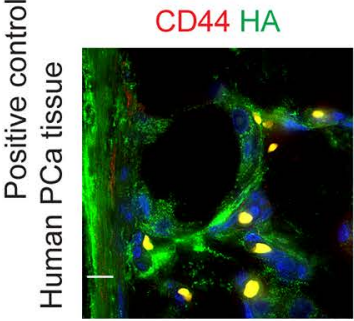

$\alpha 6$ integrin

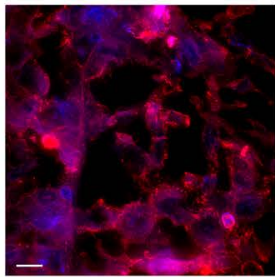

$\alpha 6$ integrin

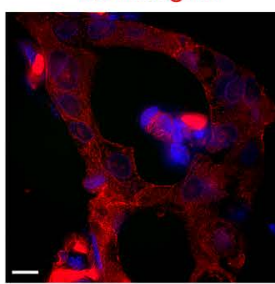

ECadherin

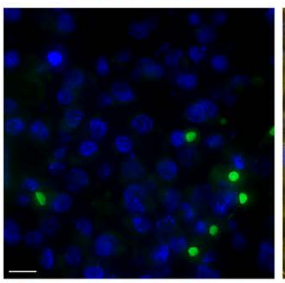

Desmin ECadherin

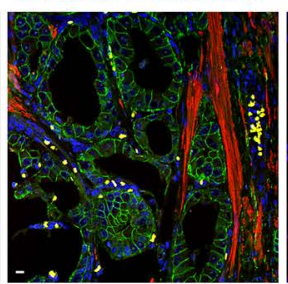

CK18 CK5/14 Desmin CK14, 15, 16, 19

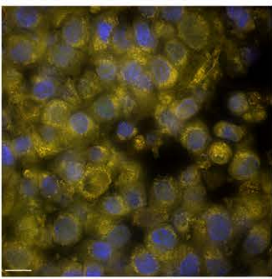

CK18

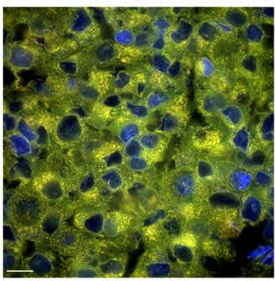

CK5/14 CK14, 15, 16, 19
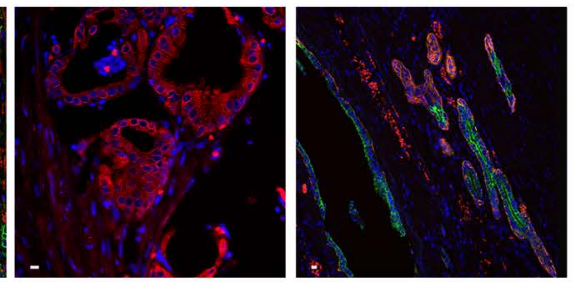